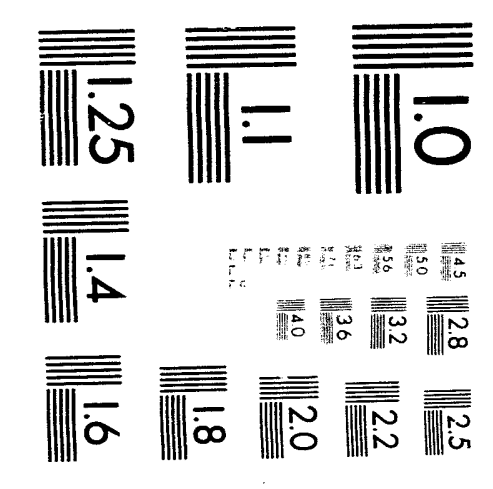



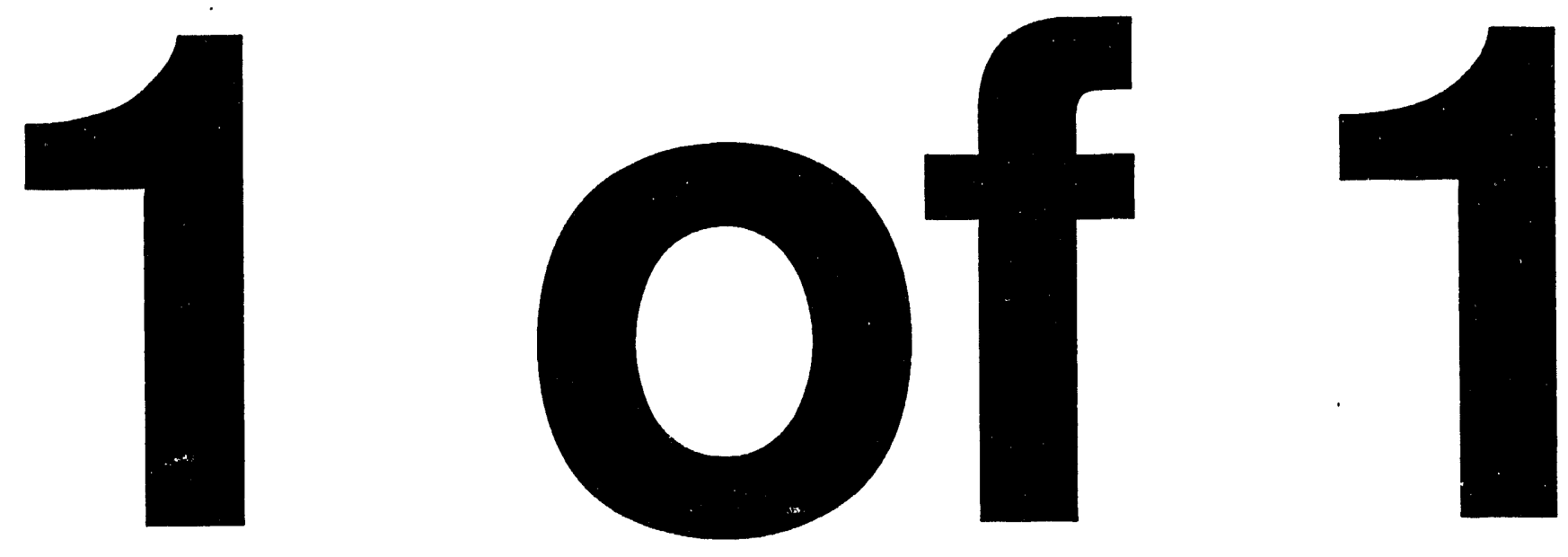
SAND93-2199

Distribution

Unlimited Release

Category UC-700

Printed September 1993

\title{
Topics in Stockpile Evaluation
}

\author{
Frank W. Müller \\ Strategic Studies Department I \\ Sandia National Laboratories \\ Albuquerque, NM 87185
}

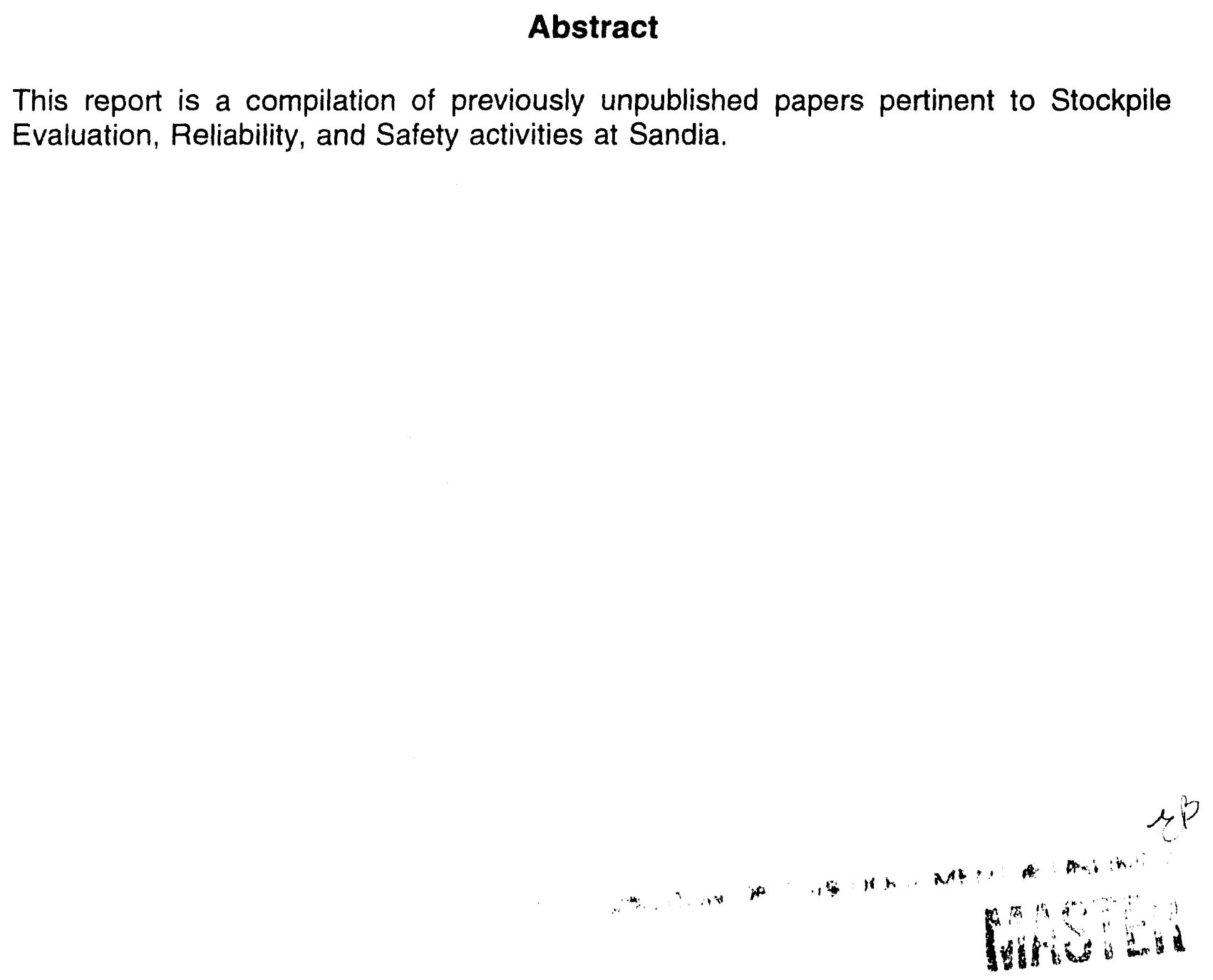


This page is intentionaily left blank. 


\section{Contents}

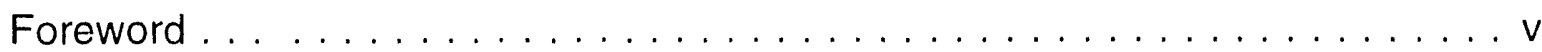

Introduction . ......................... vii

Topic I NEW MATERIAL TESTING . . . . . . . . . . . . . . I-1

Topic II "TEST SCORING" PRACTICES IN STOCKPILE EVALUATION . . II-1

Topic III CONFIDENCE LIMITS; WHAT THEY ARE, AND AREN'T . . . . . III-1

1-1 Statistical Estimation . . . . . . . . . . . . . . . . III-2

$1-2$ Practical Issues . . . . . . . . . . . . . . . . . III-5

1-3 Diversified Testing $\ldots \ldots \ldots \ldots \ldots \ldots \ldots \ldots \ldots \ldots \ldots$.II-6

1-4 Random Sampling ..................... III-9

1-5 Time Invariance . . . . . . . . . . . . . . . . . . III-10

1-6 Classification of Test Results . . . . . . . . . . . . III-10

$1-7$ Conclusions ........................ . III-10

Topic IV SAMPLE SIZES FOR 90/95 AND 90/90 SAMPLING LEVELS . . IV-1

1-1 Background ........................ IV-1

1-2 Mathematical Considerations ................. IV-3

1-3 Discussion ........................ IV-5

1-4 References ...................... IV-6

Topic $V$ PREDICTIONS INVOLVING SMALL PROBABILITIES $\ldots \ldots . V$ - 1

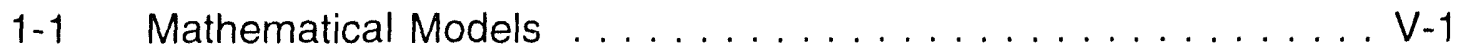

$1-2$ Probability Models $\ldots \ldots \ldots \ldots \ldots \ldots \ldots \ldots \ldots, \ldots \ldots, \ldots \ldots$

$1-3$ Conclusions ......................

Topic VI NOTES ON RELIABILITY ASSESSMENT $\ldots \ldots \ldots \ldots \ldots$. VI- 1

1-1 Reliability Modeling ................... VI-3

1-2 Reliability .......................... . .

1-3 Assessing Reliability ................... VI-11

1-4 References .......................... . . . 15

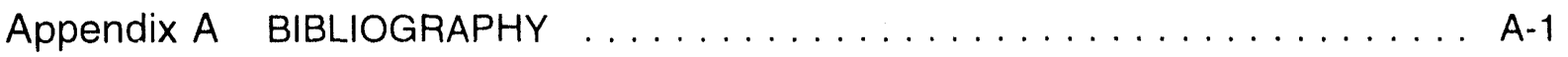

\section{Figures}

III-1 Binomial Distributions $(p=.05, .10$, and .20 for a sample of 11$) \ldots \ldots \ldots$ III-2

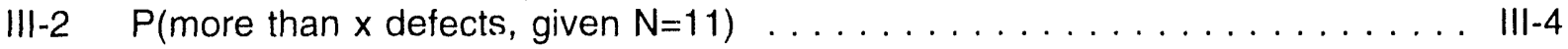

III-3 Operating Characteristics for Samples of $\mathrm{N} \ldots \ldots \ldots \ldots \ldots \ldots \ldots \ldots \ldots \ldots \ldots$

III-4 Probabilities of Observing Defectiveness $\ldots \ldots \ldots \ldots \ldots \ldots \ldots \ldots$ III-7

VI-1 Three-Component System $\ldots \ldots \ldots \ldots \ldots \ldots \ldots \ldots \ldots \ldots \ldots$ VI-5

VI-2 "Success" Regions . . . . . . . . . . . . . . . . . . . . . VI-8

VI-3 Success Region (Specification Rectangle) . . . . . . . . . . . . . VI-9

VI-4 Specification Rectangle Established so that Component Success Implies

System Success . . . . . . . . . . . . . . . . . . . . . . . VI-9

VI-5 Specification Rectangle Established so that Component Failure Implies

System Failure .......................... VI-10

VI-6 Specification Rectangle Established so that Component Failure Implies

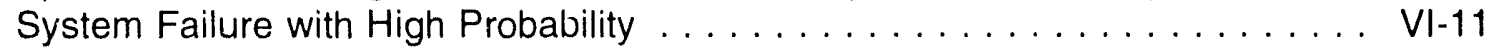


This page is intentionally left blank. 


\section{Foreword}

When a valued employee retires after 30-plus years of service, they take with them a vast store of knowledge and insights that can only be gained through hands-on experience. This is even more true for Frank Müller. He has been one of the most widely known and respected leaders and analysts in the Stockpile Evaluation community. As such, he has published a large body of reports and has edited and contributed to many more. This report is an attempt to document some of the experience and views that have not been captured in his other work.

Garry S. Brown

Department Manager, Strategic Studies 
This page is intentionally left blank. 


\section{Introduction}

It was suggested to me that papers, studies, articles, etc. on stockpile evaluation and related reliability and safety issues that I have prepared during my employment with Sandia might be of some value to future decision makers. With that hope, I have compiled this document. The information I have chosen to include has been taken from a few documents, papers, memos, etc., that were not published as formal reports (SAND documents, Tech Memos, etc.), but which address issues that remain current. I have also included an annotated bibliography of my formal reports. 
This page is intentionally left blank. 


\section{Topic I}

\section{NEW MATERIAL TESTING (JULY 1993)}

The first system evaluation programs for stockpiled nuclear weapons began in 1957. They involved sampling the inventory on a periodic basis and subjecting the sample weapons to functional tests. A year's sample represented a "snapshot" of the stockpile at a point in time, and consisted of weapons of various ages.

The New Material program began in 1959; it was originally administered by the Manufacturing Development organization as a production test program and was later absorbed into the QA program. In this program, two weapons were tested from each month's production, regardless of production quantities. (The highest fraction of units tested corresponded to "trickle" production, which was deemed appropriate.) The purpose of the tests was to provide for the prompt detection of defects and to establish a baseline for measuring aging trends in stockpiled material.

As data from the QA program accumulated, it became clear that most of the defects appearing in weapons were built into the weapons when they were new, and could be detected more promptly by increasing the new material program, while simultaneously reducing stockpile testing. A 90/95 sample was taken from the first year's production, with $2 / 3$ of the sample taken during the first half of the first cycle. Originally, new material testing was terminated after the first year, which was thought to have provided enough time for the production process to be adequately "debugged." Continuing experience with stockpile testing soon showed this idea to be wrong; process changes occurring throughout production resulted in new types of defects not present in the early production. As a result, new material testing was extended throughout production, but at a low rate of six weapons a year.

Continuing analysis of data indicated that additional new material testing after the first year would be desirable. A $90 / 90$ sample from each year's production replaced the previous rate of six per year; this doubled or tripled the sampling rate after the first year and left the first year sample intact.

When long production runs became more common, some running for more than 10 years, DOE became concerned that the new material sample rate was excessive. The primary reason the rationale was originally adopted was to discourage long production runs; severe penalties were exacted if production was extended. The desire to stretch out production won out, however, and a reexamination of the rationale was undertaken.

The concern about long production runs derives from the belief that there is an optimum production rate for any given process, and that by either stretching production out or compressing it would result in increased opportunities for new types of defects to find their way into the product. Thus, if the usual production rates were anything close to optimum from a quality 
viewpoint, quality would suffer if production was stretched out. The question that needed to be answered was "how much additional testing would be required to match the anticipated increase in defect types?"

One school of thought was that the test rate should be based on the requirement, which didn't change when the production schedules were lengthened. Proponents of this view proposed a total new material sample that was independent of the length of production; a $9(0 / 95$ quantity based on the total production quantity. This view was discarded as too optimistic.

The other school believed that sampling rates should be related to the expected frequency of defects, since the primary purpose of the program is to find and eliminate defectiveness. However, under this theory, the existing rationale suggested that the number of defect types would increase almost linearly with increased production time. No one believed that to be the case either, but there is little empirical datil on the issue of how the rate would change with changing production times.

Consequently, it appeared that a sample independent of production length would be too low, a sample linearly related to production length would be too high, and the "right" sample size would be somewhere in between. That is, if $N$ is the $9(2 / 95$ sample for the total quantity of production, $T$ is the length of production in years ( $T \geq 1$ ), and $x$ is the optimum value, then

$$
\begin{gathered}
\mathbf{N T}^{\prime \prime}=\mathbf{N} \leq \mathbf{N} \mathbf{T}^{\mathbf{x}} \leq \mathbf{N} \mathbf{T}^{1}=\mathbf{N T}, \\
\text { or } \mathbf{0} \leq \mathbf{x} \leq \mathbf{1}
\end{gathered}
$$

Without an informed choice, $x=1 / 2$ was chosen as a compromise between the two extremes, so the present rationale specifies a sample based on the formula $\mathrm{NT}^{1 / 2}$.

The sample is distributed randomly over production. The same sampling rate applies after the first six or eight months, but a higher, and variable, rate is used during the first half cycle. This higher rate averages three times the later rate to match the observed ratio of appearances of new defect types, and gradually reduces from a maximum rate of one in every two units accepted, to the lower rate specified for the remaining production.

A computer program called SAMSEL was developed to predetermine which weapons would be new material sample units. It specifies the sequence numbers of the sample weapons, where the sequence is the order in which weapons are accepted by DOE. (Since DOE accepts weapons from two sources, new production and weapons rebuilt after testing, knowing the sequence number will not permit identification of precisely which weapon will be tested until the time it is presented for acceptance. There is no way to control the order in which the weapons appear for acceptance from these two sources.)

A minimum sample of six is required for any 12 month period to guard against the potentially bad effects of reduced or sporadic production rates. 
Flight testing, part of the new material program since the 1970s, amounts to about one flight unit in every six new material sample units. (New Material Flight Tests (NMFTs) differ from the original Stockpile Flight Tests (SFTs) in that the warheads have never been associated with missiles. Because coordination of these flights is harder than for SFTs, and Service support for them is less, the number of NMFTs has been less. The present rationale, with its explicit one-in-six requirement, has made coordination somewhat easier.

Savings offered by adopting the current rationale varied from a few percent for small production quantities completed in three years, to over $40 \%$ for large production quantities produced over ten years.

In the late 1960s a number of changes to the New Material and Stockpile Evaluation Programs (NMSEPs) were made in response to observed test results. An important change was the introduction of Accelerated Aging Units (AAUs) to provide early aging information on the properties of the combinations of materials in weapons. One or two weapons were scheduled-in addition to the $\mathrm{NT}^{1 / 2}$ quantity - during the production period as AAUs. These weapons were, and are, typically exposed to a year of temperature cycling within the limits of the Stockpile-to-Target Sequence (STS) and then functionally tested in the same manner as any lab test unit. 
This page is intentionally left blank. 


\section{Topic II}

\section{"TEST SCORING" PRACTICES IN STOCKPILE EVALUATION (MAY 1993)}

"Scoring" of tests of NMSEP weapon samples is obscure in the DOE system. Scoring of system tests has been used by the Services for a long time in conjunction with their reliability assessment process. Flight tests have traditionally formed their basis for reliability assessment; lab tests have played a subordinate role, looking for trends, etc., but are not normally used directly in the assessment process.

The DOE reliability assessment process is quite different from DoD's. Before stockpile evaluation programs for sealed pit weapons were undertaken, there was little functional testing of weapons at a "system" level. Reliability assessment was based on component-level testing, using "applicable data" from any source. Scoring was not even possible at the systemlevel-most data were taken on tests of unassociated components (i.e., components which had no system identity). Consequently, all scoring was considered in relation to components, and the tests were recorded in data bases for each component. Assessments of system performance were obtained by using mathematical models to combine assessments for the individual components.

The same component-level reliability assessment process is still used today by reducing system test results to the component level. However, scoring at the weapon-level began when modern stockpile evaluation programs were initiated in the late 1950s. These programs were conducted by a Quality Assurance Agency (QAA); an independent, almost isolated Sandia organization charged with assuring DOE (then AEC) that weapons were reliable and safe. The QAA initiated system-level testing of new and stockpiled weapons, but did not assess reliability - this would have meant using data from tests specified by the project groups (horrors!) unless the reliability assessment process was changed to be more like that of the Services. Rather than do that, the QAA invented another (independent) measure, called "functionability," which, in reality, was the probability of passing a laboratory system test. It was based solely on the QAA's system lab tests, which were all alike so valid confidence statements could be made. To accomplish this, each sample weapon had to be "scored" in terms of functionability.

At the time, the reliability organization was basically part of the design team (which is why they were not included in the QAA). The last reliability assessment published was in the Final Development Report near FPU. The oft repeated explanation by the reliability organization for this practice was that there was no reason to change the assessment; if a problem was observed, it would be corrected and would restore the reliability to the level reported in the Final Development Report!

When flight testing was introduced as a separate part of the Stockpile Evaluation Program (circa 1963), the QAA readily accepted the Service request that we score flight tests in Quick Look Reports which were issued shortly after each flight test. We were used to scoring the lab tests, so flights posed no problem. However, confidence statements made by DOE were 
restricted to functionability, as measured by lab testing. (It was recognized that differences in lab and flight testing would jeopardize the validity of confidence statements.) Scoring of flight tests was only of modest interest to DOE because annual test quantities were so small. (Accumulating data over time was not done for confidence determinations, though it was done circumspectly - at the component-level-as part of the reliability assessment process.)

Functionability statements were discontinued altogether in the mid 1960s when it was decided that the reliability assessment would be offered as the DOE's best statement of reliability, and, like before, would not include confidence limits. A continuing reliability assessment program was initiated that would track weapon reliabilities until weapon retirement. This gave birth in 1965 to the DOE Weapons Reliability Report. Functionability statements were considered a confusion factor, and were discarded. (Until a few years ago, the QAA, or its successor organizations, published the DOE (then AEC) Weapons Reliability Report, generally using assessments provided by the reliability organization. The reliability organization is now considered to be sufficiently independent of the design group to publish the report themselves.)

In about 1970 the Stockpile Evaluation Program was modified so that a single sample of weapons was provided for both lab and flight testing. Also, further diversification of both lab and flight testing was introduced, which reinforced the drift away from confidence statements; no longer could anyone imagine that all tests were alike. With this shift in philosophy, weapon scoring became something of an anachronism, except for the continued need to provide scores to the Services for flight tests. The extension of scoring to present day tests is largely a carryover from these earlier times. It is confusing in today's context because of the diversity of testing. The following discussion addresses the effect of diversified testing on scoring.

First, there are probably as many reliabilities for a weapon as there are combinations of use factors such as options, applications, launch platforms, and use conditions (environments) specified in the STS. Any single lab test provides information about one combination of use factors, and the performance of the hardware tested may be scored relative to it. However, in the laboratory, "secondary" tests are continued until all the weapon's hardware is evaluated against some combination of the above use factors (e.g., after replacing or simulating hardware destroyed in the first, or "primary," test, hardware used in another option can be evaluated.) Information gleaned from these secondary tests pertains to different combinations of use factors, hence to potentially different weapon reliabilities. Since it is possible that a weapon could operate successfully in one combination and fail in another, scoring on the basis of a single test is ambiguous.

In the data banks, weapon failure is conservatively scored if there are one or more combinations of use factors in which weapon performance was unsuccessful; i.e., the weapon is scored a success only if all primary and secondary tests were successful. By this definition, a weapon can be scored accurately after the primary test only when a weapon failure occurs; a success can be countermanded if we later detect a (weapon) failure in one of the secondary tests. 
As implied earlier, none of these weapon-level considerations has any direct effect on the reliability assessment process. Regardless of a weapon's score, any defect observed is investigated individually. Once the cause of a defect is understood and scored at the component level, reliability analysts collect all the existing data on the culpable component(s), and determine which of these data are applicable to the particular failure mechanism involved. When appropriate, revised component assessments are then substituted into the math model and a new weapon assessment is computed.

We use the scores of tests to compute the average failure rate of all test weapons; this is a crude way of evaluating both reliability and the reliability assessment process. The failure rate is conservatively high for a couple of reasons.

a. Tests are generally performed under worst case STS conditions in which reliability is expected to be lowest, and

b. Many test weapons labelled as failures would have, or did, perform successfully in some combinations of use factors.

It is therefore gratifying, if not surprising, that the average failure rate of all weapons tested is as low as it is (between one and two percent), comparing favorably to averages of both allowed (per MC requirements) and assessed failure rates.

To conclude, scoring today is mainly of interest to DoD. Within DOE, it serves only to provide a rough idea of how well the reliability assessment process tracks true reliability, but does not affect the assessment process directly. For planning purposes, the reliability assessments provided in the DOE Weapons Reliability Reports and Joint DoD/DOE Reliability Reports should be used. 
This page is intentionally left blank. 


\section{Topic III}

\section{CONFIDENCE LIMITS; WHAT THEY ARE, AND AREN'T (JUNE 1993)}

Many people, both in DoD and DOE, have become accustomed to references to "Confidence Limits." They may even refer to them themselves in discussions about sizing test programs to "demonstrate" reliability. However, relatively few are steeped enough in the lore of classical mathematical statistics to understand Confidence Limits and, unfortunately, most of the people controlling our budgets and programs must be counted among this latter group. Maybe even you are in it! Let's see!

What does the following statement mean?

"DOE has demonstrated, with $90 \%$ confidence, that this weapon's reliability is at least 90."

For the uninitiated, the best recourse is to appeal to the dictionary. Confidence, according to the dictionary, means "trust." Demonstrate means "to prove or make evident by reasoning or adducing evidence."

Ergo, the statement must mean that DOE says they can place almost complete trust (i.e., $90 \%$ ) in the assertion that this weapon's reliability is at least .90 . Right?

\section{WRONG!!!}

"Confidence" in the reliability of DOE weapons depends on much more than statistical procedures. There is nothing mathematically wrong with the statistical procedures. They do provide a way to deal with statistical sampling uncertainties arising in ideal sampling situations. In the context of DOE weapons, however, the reliability assessment process and the test programs supporting it are far from ideal. There are innumerable non-statistical factors which influence the sampling and estimation process. These factors add uncertainties which are not, indeed cannot be, measured. And the resulting uncertainties can-and often do-completely swamp the statistical uncertainties! Thus calling these intervals "confidence" (read "trustworthy") intervals conveys a false impression that all uncertainties have been taken into account. The situation is somewhat analogous to marketing a cake as "low calorie," knowing that the claim does not include the frosting. It is disingenuous.

In what follows, we consider statistical estimation, including both point and interval estimation, in an ideal statistical framework. We then examine what happens when we address the practical reliability and surveillance testing issues. We conclude with some suggestions as to how we might avoid the semantic difficulties associated with the terminology of confidence limits. 


\section{1-1 Statistical Estimation}

The classical theory of statistical estimation involves estimating population parameters from tests or inspections of samples. Most people are familiar with the simple point estimate of $\boldsymbol{x} / \boldsymbol{n}$ to describe the fraction of the sampled population having a given attribute, where $\boldsymbol{x}$ is the number of sample units having that attribute, and $\boldsymbol{n}$ is the sample size. Confidence limits are another type of estimate. Loosely speaking, under ideal circumstances they describe the statistical precision of the point estimate.

Consider the simple sampling process of drawing colored balls from an urn. Here estimation is about as pure as it can be. A sample of balls is drawn at random from the urn and it is noted whether the ball is, say, red or blue (data). The fraction, $\boldsymbol{x} / \boldsymbol{n}$, of red balls $(\boldsymbol{x})$ in the sample $(\boldsymbol{n})$ is then taken as the estimate of the fraction of red balls that were in the urn. To get some idea of the precision of the estimate, we establish confidence limits.

In a sample of $n$ balls, any value of $i, i=0,1, \ldots, n$, red balls is possible. We can compute the probability distribution for $i$ if we postulate the fraction, $p$, of red balls that actually existed in the population; each postulated fraction produces a different probability distribution. These distributions are more or less centered around $\boldsymbol{p}$; the fraction of the sample that is red would most often tend to be close to the population value, with widely disparate fractions occurring infrequently. Figure III- 1 shows the probability distributions for $i$ when $p=.05, .10$, and 20 , and when $\mathrm{n}=11$, the current stockpile sample size.

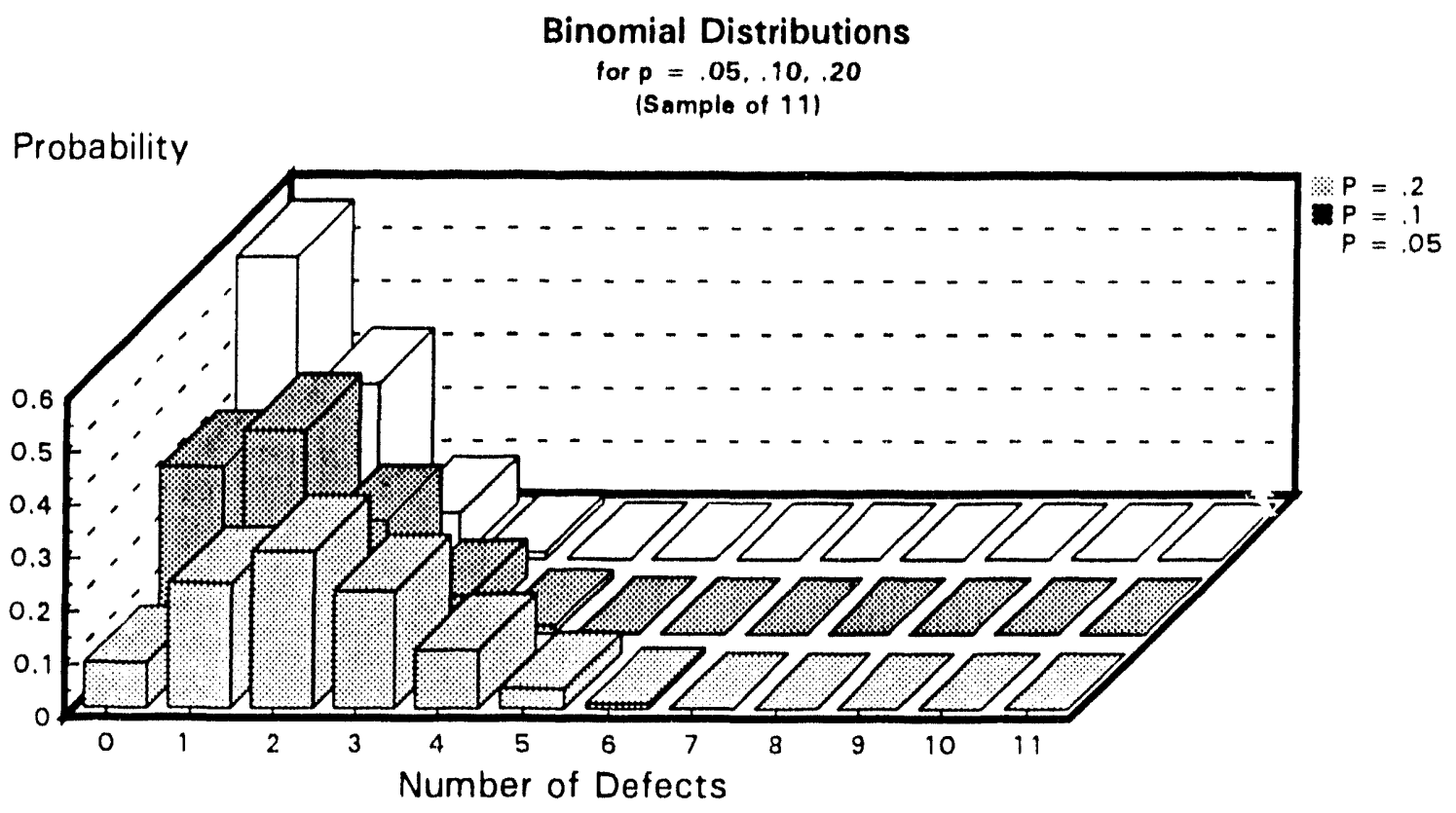

Figure III-1. Binomial Distributions $(p=.05, .10$, and .20 for a sample of 11$)$ 
By using such distributions, a "system" of confidence intervals can be constructed so that, for each possible sample result, an upper confidence limit (UCL) on the fraction of red balls is established. (We take the lower confidence limit (LCL) to be zero.) By using the number of red balls, $i$, to order the sample results on a "good/bad" scale, we can produce a system of confidence limits. In this system, if red is "bad," the "best" sample result ( $i=0$ ) gives the lowest limit, the next best result $(i=1)$ gives the next lowest limit, etc. That is, we want to establish a set of $L_{i}$ such that

$$
0 \leq \mathrm{L}_{0} \leq \mathrm{L}_{1} \leq \mathrm{L}_{2} \ldots \leq \mathrm{L}_{\mathrm{i}} \ldots \leq \mathrm{L}_{\mathrm{N}} \leq \mathbf{1 . 0}
$$

When a sample result is observed, the number, $i$, of red balls in the sample dictates which of the $L_{i}$ is to be taken as the upper confidence limit.

We can establish the $L_{i}$ as follows. To establish $L_{0}$, assume that $p$ is actually between $L_{0}$ and $L_{1}$. This would mean that all the intervals except the one bounded by $L_{0}$, the limit associated with no red balls, contain the true value of $p$. We want to restrict the (pre-sampling) probability of obtaining the sample result of no red balls; the "confidence coefficient," $\alpha$, is the restriction. That is, the probability of observing no red balls is to be no greater than $\alpha$ when $p$ is between $\mathrm{L}_{0}$ and $\mathrm{L}_{1}$, or, equivalently, we want the probability of seeing one or more red balls to be at least $(1-\alpha)$ when $p$ is between $L_{0}$ and $L_{1}$. (The value $(1-\alpha)$ is called the confidence level.) To get the shortest confidence intervals possible, we seek the smallest value of $p$ satisfying this condition, namely the value where the probability of no red balls is equal to $\alpha$. For the binomial distribution, this gives us the following relation.

$$
(1-\mathbf{p})^{n}=\alpha, \text { from which } \mathbf{p}=1-\alpha^{1 / n}=\mathbf{L}_{\mathbf{i}}
$$

$L_{1}$ is obtained by now assuming that $p$ is between $L_{1}$ and $L_{2}$, and proceeding in similar fashion. Now there are two sample results which will produce confidence intervals that do not contain $p$, so the probability of seeing zero or one red ball is set equal to $\alpha$. That is,

$$
(1-p)^{n}+n p(1-p)^{n-1}=\alpha .
$$

This (and the equations for the remaining $L_{1}$, determined by successively moving the assumed position of $\boldsymbol{p}$ relative to the $L_{1}$ and each time adding another sample result to those which produce intervals that won't contain the true value of $p$ ) cannot be solved in closed form for $p$, but can be solved graphically. Figure III-2 shows plots of the probabilities of observing more than 0,1 , or 2 red balls in a sample of 11 , for different $p$. The first few values of $L_{i}\left(L_{0}\right.$, $L_{1}$, and $L_{2}$ ) can be read directly from these curves for any value of $(1-\alpha)$. For instance, $90 \%$ UCLs (i.e., $100(1-\alpha) \%$ UCLs) can be read on the $x$-axis where the curves intersect the 9 level; $80 \%$ UCLs from where the curves intersect the .8 level, etc. The $90 \%$ UCLs $\mathrm{L}_{0}$ and $\mathrm{L}_{1}$ are about .19 , and .31 , respectively. $\mathrm{L}_{2}$ is off-scale, but would be about 4 . The $80 \%$ UCLs are about $.14, .25$, and .35 , respectively. 


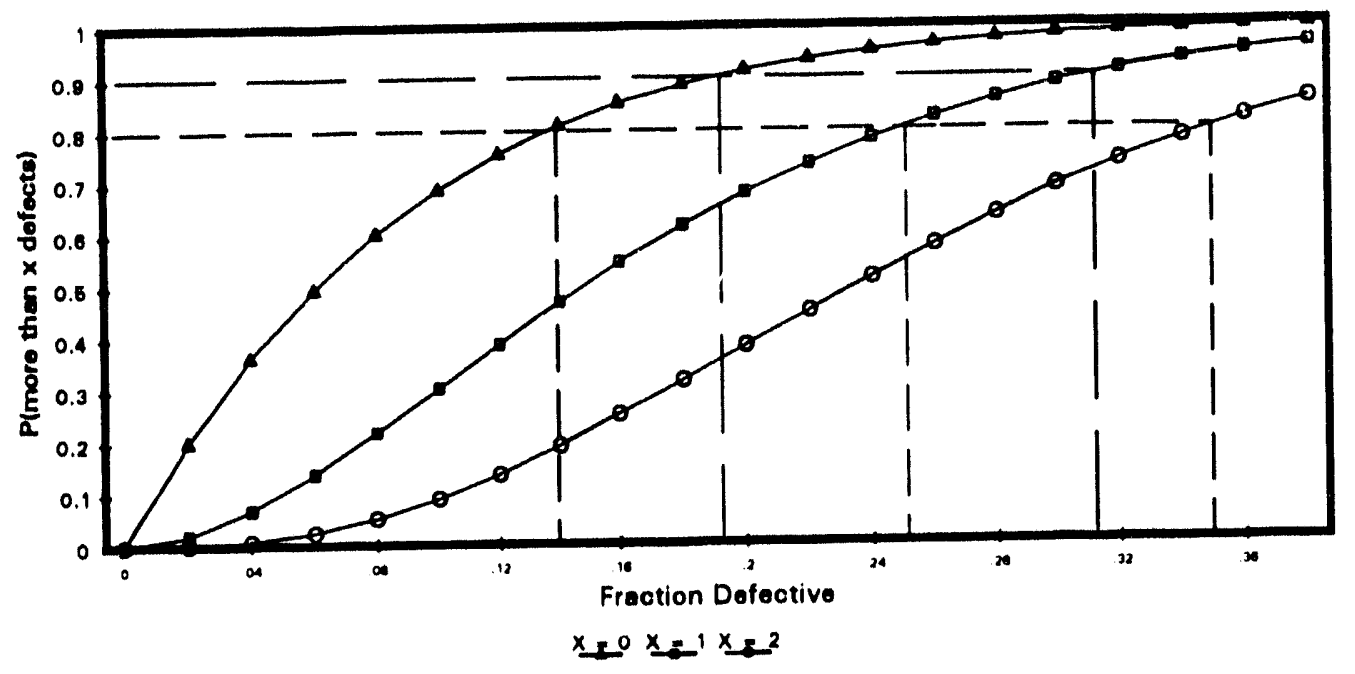

Figure III-2. $P$ (more than $x$ defects, given $N=11$ )

If, in a long series of random samples of 11 balls per sample, the appropriate $\mathrm{L}_{\mathrm{i}}$ are taken as $100(1-\alpha) \%$ UCLs for the successive sample results, approximately $100(1-\alpha) \%$ of those limits would contain the true fraction defective. Of course, a particular limit either does, or does not, contain the true fraction; we only know that before the sample was drawn, the probability was $1-\alpha$ that the limit would contain it.

To establish, say with $90 \%$ confidence, that the fraction of red balls is not greater than some prescribed value of $\boldsymbol{p}$, the sample size can be adjusted. Figure III-3 shows the probability of seeing no defects in samples of 11,22 , and 44 for different fractions defective. Curves like these will yield the smallest sample size that can produce the desired statement. We would seek the sample size for which, with the desired value of $\boldsymbol{p}$, the probability of observing no red balls was $\alpha=.10$. Then, if no red balls are observed in the sample, we can make the statement that the fraction is not greater than $p$, with $90 \%$ confidence. For instance, to establish, with $90 \%$ confidence, that the failure rate was less than .05 , a sample of 45 will suffice if no red balls are observed.

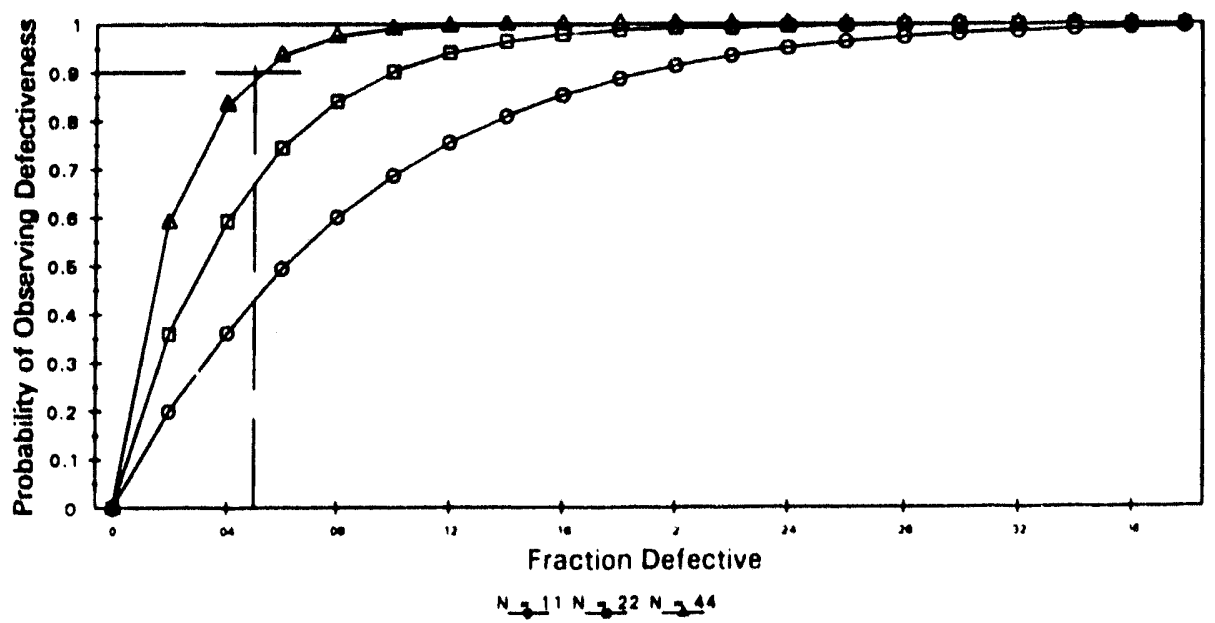

Figure III-3. Operating Characteristics for Samples of $N$ 


\section{1-2 Practical Issues}

Estimating the fraction of red balls in an urn is one thing; estimating (assessing) the reliability of DOE weapons is quite another. To begin with, there isn't just one reliability of a weapon. Weapons are to be used in different environmental conditions, with different fuzing and delivery options, different delivery systems, etc. Reliability is a function of both hardware and the way it is used, so different reliabilities can exist for each possible combination of the above conditions ("use factors"). DOE tries to determine the minimum reliability in that set of reliabilities which, collectively, describes the behavior of the weapon in all of its required use conditions. But determining that reliability requires knowledge of all the other reliabilities; how else would we know which was the minimum?

Next, unlike the urn example, one doesn't just select a sample of weapons to determine reliability and note whether they are good or bad. We conduct functional tests to score weapon or component performance. These tests are destructive to many of the components, and are very expensive. This places real constraints on the amount of testing that can be done. To illustrate, if a sample of 50 were considered adequate to estimate reliability for one combination of use factors at a point in time, and we identified a dozen combinations of the above factors that corresponded to worst case reliabilities, 600 weapons would have to be destroyed to provide current estimates of these reliabilities. The issue would then arise as to when we would have to repeat the testing, because, as we know, reliability changes with the age of the weapons! Not only would the costs be exorbitant, hut we would have to build many more weapons for testing than we would ever need for war!

Contrast that with the stockpile sampling rationale of today, which specifies a sample of about 11 weapons a year, where the weapons are allocated among a number of different kinds of tests (addressing different combinations of use factors), both in the laboratory and in flight. When judged appropriate, this information is combined with that from system and component testing conducted during development (to establish and verify design capability), and during production (for product acceptance) to increase the amount of information available for assessment. New Material and Stockpile Evaluation testing complements the earlier testing, and serves to validate the data collected in those tests.

Finally, all tests simulate, to one degree or other, the conditions expected if the weapons were to be used in anger. The tests involving the least simulation are flight tests; the most simulation is associated with component testing. In any event, engineering judgment must be applied to each test to decide whether its results really measure performance in actual use.

The point of this discussion is to allude to the complexity of the reliability assessment process and its extreme dependency on experience and engineering judgment, which is always subject to error, sometimes colossal error. These errors can dramatically affect the reliability assessments and confidence limits, and represent non-statistical uncertainties not addressed by confidence limits. We have little choice but to make assessments and use them as guides to actions and decisions. Perhaps a saving feature is that reliability assessment is a continuing 
process in which significiant errors will eventually be discovered and the assessments corrected, at least for those combinations of use factors which are being tested. But we don't have to quote confidence linits, implying that all uncertainties have been accounted for by using such words as "confidence" and "demonstration." At best, confidence statements are tautologies ("If ...then" statements) in which the premises are false. They are about as useful as statements like "If wishes were horses, then beggars would ride." At worst, they are gross misrepresentations of the state of our knowledge about reliability.

The next few sections describe the more serious problems with the application of confidence limits to the DOE assessment process.

\section{1-3 Diversified Testing}

The most conspicuous problem with trying to establish confidence limits on reliability is the diversified testing. To provide a statistically valid estimate, the tests whose results are pooled must be equally effective in discovering defects. One way to ensure this is to make all the test conditions identical from one sample unit to the next. This was done in the first stockpile evaluation programs; all tests were under ambient conditions in the laboratory, which provided some information about one of the reliabilities of interest, but unfortunately one that would be expected to be among the highest in the set.

If we knew which set of conditions would result in the lowest reliability, we presumably could test all units under those conditions. But we don't, and can't, know which set of conditions leads to the lowest reliability until we test under a variety of conditions. Further, we kept finding failures that didn't appear in our test program; we found them instead in retirement, military inspections, special tests, etc. As a result, we diversified the tests to increase the number of defect lypes visible to the test program, and to satisfy ourselves that we are addressing reliability under conditions that are at least close to being worst case conditions.

The decision to diversify testing, though necessary, has made reliability assessment and test planning very difficult. Reliability assessment is performed at the component level, with all the component assessments combined in a math model to produce an assessment, or assessments, for the weapon. The analyst gathers all the data on tests of the component, judges which of these data are applicable to the conditions of interest, and combines the adjudged applicable data to produce an assessment. Having made the judgments about applicability, the assessment is the simple $\boldsymbol{x} / \boldsymbol{n}$ discussed carlier.

One problem that exists is that certain defects can cause failure in one kind of test but not in another, or they can caluse failure at a higher or lower rate in one test than they would in others. The situation is well illustrated by an experience with the W62, a warhead for MMII. The first NMSEP failure observed was the fifth llight test unit. An environmental sensing device (ESD) (integrating accelerometer) failed, but, because the component could not be recovered for post mortem evaluation to determine the precise cause of the failure, little beyond that simple fact wals known. 
Several hundreds of these ESDs had been subjected to static weight tests during production with no failures, and approximately 60 systems had been dynamically tested in the laboratory, again with no failures. The judgment was made to ignore the static tests and use only dynamic tests conducted in the laboratory and flight. This made for an $\boldsymbol{x} / \boldsymbol{n}$ of something like $2 \%$, and, if one wanted to compute it, an upper $10 \%$ confidence limit of about $6 \%$ for failure rate.

It was later discovered that, because of the steep acceleration ramp used to simulate the "worst" acceleration, this particular defect would not cause failure in laboratory testing, so none of the laboratory tests were applicable! The only tests known to be applicable at the moment were the flight tests, which by that time had increased in number to two failures in 15 flights, yielding a failure rate of $13 \%$ and a $90 \%$ upper confidence limit of something like $32 \%$ ! How much trust would you place in the $90 \%$ confidence limit of $6 \%$ ?

The continued appearance of defects that were only visible in lab or flight tests has been studied. Approximately one in every tive defects observed in warhead flight tests was judged "flight-unique" (could only be observed in flight). A similar result was found with "lab-unique" defects. For a sample of 22 weapons, of which eight were flights, the actual sample sizes for the three different kinds of defects are as follows.

$\begin{array}{lc}\text { Persistent (visible in either test) } & 22 \\ \text { Flight-unique } & 8 \\ \text { Lab-unique } & 14\end{array}$

Figure III-4 plots probabilities of observing defectiveness in the sample of 22 versus failure rate for two cases. The top curve considers that any defects would be "persistent," i.e., detectable in either kind of test. If no defects were observed. the $90 \%$ UCL would be .10 , the usual statement based on the $90 / 90$ criterion. $(90 / 90$ is the conventional way of referring to a \%)\%LCL for reliability.)

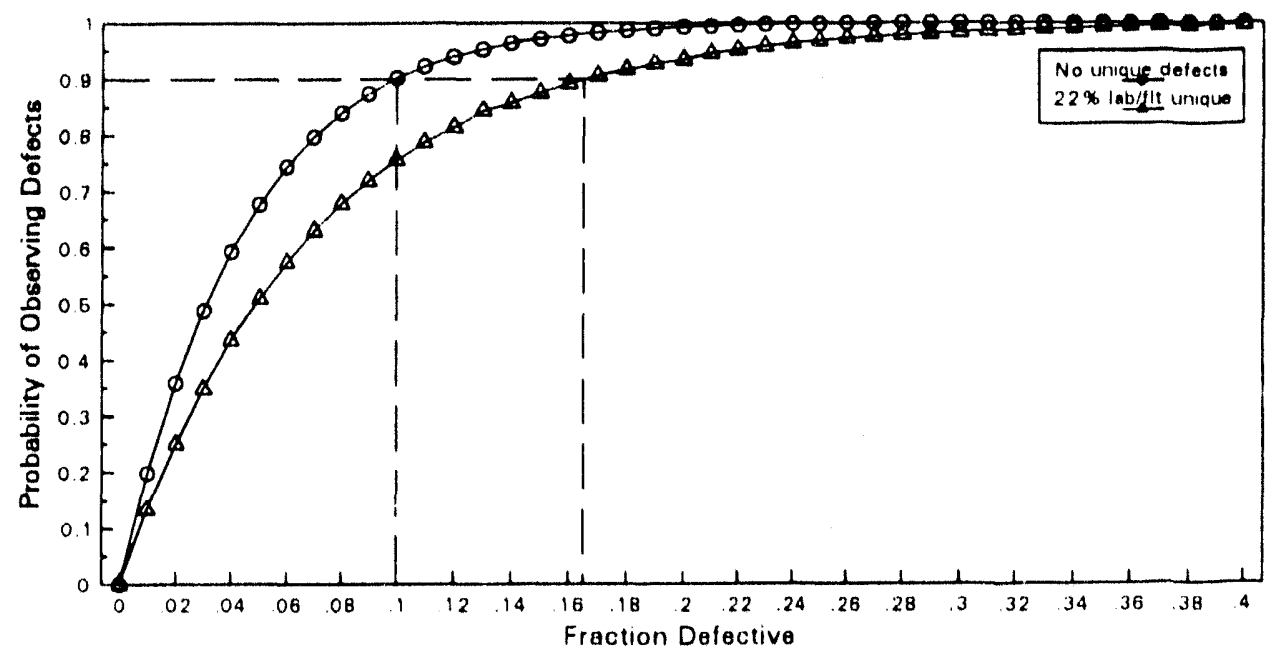

Figure III-4. Probabilities of Observing Defectiveness 
However, because of the presence of test unique defects, a different approach must be taken. Here the three categories of defect (persistent, lab, and flight unique) are treated separately, with each type of defect considered in relation to its sample size. The results are then combined by weighting each result by the probability that a defect will belong to that category. For instance, if probabilities of each of the two kinds of unique defects were .22, then the "true" $90 \%$ confidence limit would be around .165. (The bottom curve in Figure III-4 is based on eight flights in a sample of 22.)

The frequencies of unique defects are not known, but must be estimated or postulated, admitting arother source of uncertainty. The above probabilities of .2 appear to be about right (for warheads), but this is based on the aggregate experience with many types of weapons (to get enough data points), so again questions of applicability can be raised.

The situation gets worse. If placing defects in the correct categories (so the category frequencies can be estimated) is difficult when only two categories of a test are considered, the difficulty becomes virtually unmanageable when one considers the actual number of test variations used to support reliability assessment. For example, many types of tests are conducted in New Material and Stockpile Evaluation Programs alone, each chosen precisely because it can expose a unique, but credible, kind of defect not visible in the other tests.

In general, $\boldsymbol{M}$ different types of tests result in $2^{\mathrm{M}-1}$ categories of defects whose frequencies must be estimated. In one weapon program, 14 different types of test were identified in NMSEP testing alone. Without even considering additional types of tests involved in production, this results in over 16,000 different categories! These categories would ultimately be grouped by the sample size that each experiences. If each type of test was conducted at least once in a two year period (sample size of 22), this would result in 22 different groupings or sample sizes. The judgment factors come in distributing the 16,000 categories among the 22 groups, and then estimating how often each group will occur. If it were possible to do that (it isn't!), the probability of observing at least one defect in the sample of 22 would be

$$
P(\text { Defect })=\Sigma \alpha_{x}[n ! / x !(n-x) !] p^{x}(1-p)^{n-x}
$$

where the range of summation is from $\mathrm{x}=1$ to $n$. The $\alpha_{\mathrm{x}}$ represent the probabilities that a defect has a sample size of $\boldsymbol{x}, \Sigma \alpha_{x} \leq 1.0$. For at least those values of $\boldsymbol{x}$ equal to numbers tested in unique tests, the $\alpha_{x}>0$ (otherwise we wouldn't do that test).

Increasing the number of different types of tests means that there will be more of the defects associated with the smaller sample sizes, which would further degrade the confidence limit. If two different kinds of tests degrades a confidence limit as much as was indicated earlier, the degradation from all the different tests could be devastating. 


\section{1-4 Random Sampling}

Random sampling ensures that the sample is as "representative" of a population as we can make it, so that estimates based on repeated sampling will be predictably "near" the population parameters or frequencies. Consequently, we strive to use random samples whenever possible. In stockpile evaluation, for example, we resist arguments that one weapon is as representative as another. This argument arises because of desires to substitute more accessible units for those selected at random. We also resist biasing samples towards portions of the population believed to contain more defects, e.g., to the oldest items in the population. This is a well-intentioned motive, but not only biases any estimate of the population, but may bias the sampie away from more serious defects, e.g., defects caused by production changes after the oldest items were built.

Despite our insistence on randomization whenever possible, reliability assessment within DOE is forced to rely on "pooling" component- and system-level data from many sources. No amount of imagination can suppose that the collection of all these data are equivalent to a random sample from the weapon population. Tool Made Sample units are the first units built; production testing is stratified by production lots; New Material samples are selected at different rates throughout production; Stockpile samples are randomly selected from the inventory each year, but are accumulated over time (while aging may be degrading the stockpile); special tests are often of completely non-random units aimed at real or imagined problems in selected groups of units, etc.

In order to combine these data into a reliability assessment or confidence limit, we are forced to assume that the population is reasonably homogeneous; we do this in ignorance of many of the underlying ciuse/effect influences in operation. Homogeneity means that whatever properties the items in the population possess are "mixed" (randomized) throughout the population well enough that any subpopulation of reasonable size has pretty much the same characteristics as the total population. Items possessing the same properties are not clustered together by virtue of time of build, geographic location, etc. When that is the case, sample selection would not have to be random; homogeneity takes care of that for us. Heterogeneous, i.e., non-homogeneous, populations have dissimilar subpopulations; non-random samples based on time of build, geographic location, etc., are more likely to display characteristics very different from the total population than are random samples.

Past errors of judgment have shown how limited our ability is to identify and address all the myriad subtle influences that shape populations, or to understand the subtle features of homogeneity.

Unfortunately, judgments that things are mixed well enough for the population to be considered homogeneous are easily made, but are frequently wrong because they are made without understanding all the influences at work. Even deliberate attempts to mix things do not work well. For example, sequences of bridge hands "dealt" by computers are known to be significantly different from those dealt after shuffling the cards by hand; shuffling cards fails to 
mix the the way the random number generator does. To rely on adequate mixing to occur as a result of natural movements of things is inviting trouble, yet we are forced to do this to increase the dita points available for reliability assessment.

\section{1-5 Time Invariance}

The population should not change during the sampling process. This seems obvious, hut the practice of accumulating results of samples taken over a period of time is commonplace, naively ignoring aging or handling effects that can significantly alter a population. For instance, if reliability of a population of devices is high when sampling hegins and then drops while sampling is underway, the sample will not be representative of anything. Further, the early good results will mask a growing problem. perhaps suggesting that no action is needed when it is needed. A time interval of a year could he enough for product to change; in two or more years it is likely that product will change.

\section{1-6 Classification of Test Results}

In the urn example, errors in classifying the color of the balls would result in totally erroneous estimates and confidence limits. This would seem unlikely, hut what if the person noting the colors of the balls was color blind? Or, as has often been documented in Q( practice, the person doing the classification may have seen so many blue balls he has built up a mental expectation that all the halls are hlue, and literally does not "see" a red ball when it appears"?

Of course, misclassifying test results is much more likely than misclassifying the color of halls drawn from an urn. For example, correctly interpreting the meaning, in terms of system performance, of a component failing to meet its specification requires considerable engineering judgment and knowledge of system responses. Component specifications often involve "ladder tolerancing." perhaps even "over tests." suggesting that they are not a good surrogate for systemlevel criteria.

\section{1-7 Conclusions}

We make assumptions of homogeneity in order 10 do assessment. We do look for possible breaches of homogeneity-e.g. we chose not to use static test results in the W62 example earlier-hut recognize our limitations. Nonetheless, we consider our assessments the hest that can be made with our imperfect knowledge about the weapon, and offer them as guidance to DoD for their planning. It may not he very good guidance-any assessment is certainly wrong. at least 10 a degree-but it is the hest we can do. Despite the fact that confidence limits can be computed if enough false assumptions are made, we can offer no real guarantees.

The dictionary tells most people to equate confidence with trustworthiness, and the mathematical tractability of confidence limits attracts those who are seeking answers to difficult questions about how good are reliability assessments and how many weapons do we need to test. 
Confidence limits and statements have their uses, but in practical programs where relatively small quantities of diversified tests are searching for defects that can influence weapon behavior in a wide range of conditions, their use is misleading, particularly in relation to reliability assessment or reliability "demonstration." Too many non-statistical judgments (assumptions) are involved where errors can, and often do, overshadow the classical statistical uncertainties.

The above is not intended to keep people from computing probabilities of observing defects with given sample sizes. For example, a $90 / 90$ criterion specifies a sample size that has a $90 \%$ chance of observing a defect if the fraction of the population possessing that defect is at least .10, if defects are not unique to tests conducted on only a portion of the sample, if the sample is random, and if we are able to recognize a defect when we see it. Such measures are useful in test planning but, for all of the reasons mentioned earlier, they should not tempt us to make comfidence statements about demonstrated levels of reliability when no failures are observed in the tests.

Nor does this paper intend to say that we can't have confidence in our weapon stockpiles or our estimates of their reliabilities. We simply should recognize that the process rests on judgments and analyses by highly experienced professionals. In the end, the only true test of the process is a retroactive look at the record over a long period of time. We can be reasonably confident, based on testing over 13,000 weapons of 50-60 weapon types over a period of 35 years, that the process, as refined and improved through the years, has maintained the required reliabilities. 
This page is intentionally left blank. 


\section{Section IV}

\section{SAMPLE SIZES FOR 90/95 AND 90/90 SAMPLING LEVELS (AUGUST 1992)}

Sandia has provided sampling criteria for the DOE Stockpile Evaluation Programs which use so-called 90/95 and 90/90 sample sizes. What we have referred to as a 90/95 sample size is determined by finding the minimum sample size, $\boldsymbol{n}$, for which the following inequality holds:

$$
\frac{\Gamma(.95 N) \Gamma(N-n+1)}{\Gamma(N+1) \Gamma(.95 N-n)} \leq .10
$$

where $\boldsymbol{N}$ is population size and $\boldsymbol{n}$ is sample size, and $\Gamma(\boldsymbol{x})$ is a Gamma function (tabulated in most math handbooks). The expression on the left may be roughly interpreted as the "pre-sampling" probability of observing no defects in a sample taken from a population with more than $5 \%$ defective items.

The tables in the Stockpile Evaluation Manual (refer to Reference 1, page IV-6) provide the sample sizes determined thusly. 90/90 sample sizes are defined similarly and are also provided in the manual.

\section{1-1 Background}

Sample sizes have been the subject of continuing interest since the beginning of the Stockpile Evaluation Programs in the late 1950s. Since that time, annual sampling rates have been reduced by an order of magnitude, from 100 a year (two phases, or cycles, of 50 each) to approximately 10 a year (half of a 90/90 sample each year). Only about $10 \%$ of this overall reduction has been directly attributable to classical statistical considerations; the first sample sizes of 50 units per phase, or cycle (to provide a $2 \%$ resolution in estimates of "functionability"), was reduced to about 44 or 45 units (a 90/95 sample) to take advantage of a statistical "signpost" that was conveniently close to what we were already doing.

Since that time, sample sizes have been identified in terms of 90/95, $90 / 90$ criteria, etc., even though the reductions in sample sizes have, with the one exception mentioned above, always been based on analyses of: 1) test results accumulated over the years of testing, 2) defects found in activities other than the Stockpile Evaluation Programs, and 3) actions taken following the observance of defects in any activity. The reason for continuing the statistical criteria is to hold constant, in sampling from different size populations, a reasonable probabilistic demand; it has seemed reasonable to provide the same chance of finding a defect that affects a given fraction of the population, regardless of the size of the population.

It is unfortunate that the sampling criteria have been expressed this way, because the sophistication of the statistical trappings has led some people to focus on a relatively minor aspect of the sampling question, and to suppose that the choice of sample size is a great deal more precise than it is. To illustrate, except for the adjustment mentioned above, all three major 
reductions have simply halved the sample size. The first major reduction in sample size was to increase the interval hetween test phases, or cycles, to a year (or more), halving the annual sampling rate. This occurred in the early 1960s, not too long after the program was initiated; the adoption of the $90 / 95$ criteria occurred at about the same time. It was based on the observation (from the data) that degradation didn't occur so rapidly that semiannual testing was necessary.

Another major reduction in the annual sampling rate was made in 1970, when it was decided that the sampling rate could be halved again. This reduction was based on the idea that initiating a serious investigation after the first observation of a defect would make the smaller sample size equivalent, in terms of overall response, to a larger sample size where serious investigation awaited several oecurrences of a defect. The reduction was accompanied by a strengthening of the "Significant Finding Investigation" (SFI) process to make sure investigations were initialed promptly and conducted earnestly.

After making the decision to reduce, we cast about for another convenient statistical "signpost," and incorporated sampling at a 9(0/90 level, which is approximately half of a $90 / 95$ level.

The last major reductions were introduced in the early and mid 1980s, when it was decided that the sampling rate could once again be halved, initially for only the later cycles, then ultimately for all cycles. These reductions were based on the decreased frequency with which new types of defects appeared in stockpile testing. (Concurrently, New Material testing was increased due to observations that most defects found in the stockpiles were "birth" defects, i.e., defects introduced hy design and/or production.)

After this decision was made, no convenient "signpost" could be found, so the rather non-statistical designation of a $90 / 90 / 2$ level was introduced, merely specifying a sample that is half of a $90 / 90$ sample; it apparently is easy to visualize a $90 / 90$ sample accumulating every two years.

There are numerous complications in determining an appropriate sampling rationale; they go much beyond the $90 / 95,90 / 90$ criteria. Or, to put it another way, some severe, and unrealistic, conditions are necessary to validate the statistical criteria. The major conditions are that defects legitimately addressed by the criteria 1) must have rates that are time-invariant over intervals in which results are accumulated, and 2) could be detected in any of the diverse kinds of test 10 which a sample unit might be subjected.

Fortunately, many defects are such that these conditions are satisfied. But for as many defects these conditions are not satisfied; aging defects are not time-invariant, and defects dependent upon conditions external to a component or system (e.g., environment and/or system configuration) are not observable in all types of tests. These defects contribute to the fraction defective of the population, too, making it difficult to attach too much meaning to the statistical criteria. 
Incidentally, these latter defect types have had almost as much influence on the sampling rationale as the former: 1) the rates at which aging has been observed has influenced the frequency of test phases, or cycles, and 2) the observed dependencies of defects on external, and often mutually exclusive, conditions has resulted in diversification of tests to ensure that all types of defects can be observed in some kind of functional test.

\section{1-2 Mathematical Considerations}

This section of the report addresses the narrower aspect of the statistical criteria used in the sampling rationale. Specifically, it deals with the mathematics and assumptions underlying these criteria, which are subtle enough in their own right to confuse the non-initiated. For example, some have criticized the approach taken by Sandia, recommending instead the determination of sample sizes using a direct hinomial calculation,

$$
(.95)^{n} \leq .10
$$

These critics are correct in concluding that the sample sizes are not strictly conventional texthook 90/95 samples; however, the choice of the criteria was deliberate, and eliminated some undesirable features of more conventional sampling criteria.

We begin by illustrating the difficulty with using the binomial calculation; it leads to a single sample size regardless of the size of the population from which the sample is drawn. The solution to the above equation is a sample of 45 , which is difficult to draw from a population of, say, 30 units! Even when the population is larger than 45, this formula will result in much larger samples than necessary.

(Theoretically, the binomial sample is appealing in that it would characterize the process parameter, $p$, which describes the probability that the next unit built by the process will be defective. To do this, a sample must provide a certain amount of information, independently of the number of units in the population. But, as illustrated above, when the population available for sampling is too small, it is impossible to provide that amount of information. However, a realizable sample can be used to address the fraction defective of the population produced (instead of the binomial process characteristic, $\boldsymbol{p}$ ). In this case, the precision of estimation need not be so high, since there are only $\mathrm{N}+1$ possible fractions defective in a population of $N$ units; $0,1 / \mathrm{N}, 2 / \mathrm{N}, \ldots, \mathrm{N} / \mathrm{N}$. The values of $p$, on the other hand, could be anything between 0 and I.)

Sampling from small populations is characterized by using the hypergeometric distribution. However, its direct use has problems, caused by the discreetness inherent to the problem. The hypergeometric calculation involves dividing the population, $N$, into a number of good, $\boldsymbol{G}$, and bad, $\boldsymbol{N}-\boldsymbol{G}$, discrete items. The probability of seeing no defects in a sample of $\boldsymbol{n}$ items is

$$
{ }_{6} \mathrm{C}_{n} /{ }_{N} \mathrm{C}_{\mathrm{n}}
$$


where ${ }_{X} C_{Y}$ is the number of ways $Y$ things can be taken from a population of $X$ things; ${ }_{X} C_{Y}=$ $X ! / Y !(X-Y) !$

Solving for the smallest integer value of $\boldsymbol{n}$ which obeys the inequality

$$
{ }_{i} C_{n} /{ }_{N} C_{n} \leq .10
$$

provides the sample size for a $90 / 95$ sample, when $N-G$ is the smallest integer for which $(\mathrm{N}-\mathrm{G}) / \mathrm{N}$ equals, or exceeds, .05 .

A plot of sample size versus population size can be produced easily enough with this formula, but it has the disturbing property that sample size is not a monotonically increasing function of population size. For instance, a fraction defective of .05 or more in a population of 21 units requires that the population contain at least two bad items, whereas in a population of 20 units, only one bad item is needed. The sample size for the population of 20 is given by the smallest integer value of $n$ satisfying the following relation.

$$
{ }_{19} \mathrm{C}_{\mathrm{n}} /{ }_{20} \mathrm{C}_{\mathrm{n}} \leq .1
$$

A sample size of $n=18$ is required.

However, in the population of 21 , there are still the same number of good items; the unit added to the population must be defective in order for the population to be at least $5 \%$ defective. This means there are now more opportunities for drawing a bad item, so the sample required from this population is smaller. The inequality is now

$$
{ }_{19} \mathrm{C}_{\mathrm{n}} /{ }_{21} \mathrm{C}_{\mathrm{n}} \leq .10 \text {, }
$$

which yields a sample size of $n=14$.

A plot of sample size versus population size is a jagged, sawtooth plot when sample sizes are computed this way. Local maxima occur at population values one less than those for which an additional defective unit has to be added in order for the fraction defective to equal, or exceed, the specified fraction-in the case above, .05 . That is, a local maximum occurs when $N=20$ in the example; when $N=21$, a second defective unit has to be added for the fraction defective to reach, or exceed, .05 . The sample size for $N=21$ is a local minimum.

We have made two modifications to the above curve. The first, and most important, is to use a "smoothing function" which connects the local minima into a smooth curve; this is where the Gamma function comes in. A way of interpreting its use is to visualize that we could always achieve a fraction defective of precisely .05 in any population if we imagined fractional defects; e.g., a value of $\mathrm{N}-\mathrm{G}=.9$ bad units in a population of 18 would yield a fraction defective of precisely .05 . With this device, we eliminate the sawtooth effect of the curve, and produce a monotonic, increasing relationship between sample size and population size. 
The Gamma function, $\Gamma(\mathrm{x})$, is defined by an integral relationship which provides values between zero and one when the independent variable, $x$, assumes values between one and two. Tables of these values are available in most math handbooks. A recursive relationship, $\Gamma(x+1)$ $=x \Gamma(x)$, permits solution for positive values of $\boldsymbol{x}$ greater than one. Also, when $\boldsymbol{x}$ is an integer, $\Gamma(x)=(x-1)$ !; this relates the Gamma function and the factorials used in the hypergeometric calculations above. In terms of the Gamma functions,

$$
{ }_{(} \mathrm{C}_{n} /{ }_{N} C_{n}=\frac{G !(N-n) !}{(G-n) ! N !}=\frac{\Gamma(G+1) \Gamma(N-n+1)}{\Gamma(G-n+1) \Gamma(N+1)}
$$

One further modification was introduced. If the .05 fraction defective were to be considered "just barely acceptable" - instead of "just barely unacceptable"-a slight reduction in sample size is possible. This change has the effect of moving the local minima to population sizes that are each one less than before-they will now occur at values of $N$ where $95 \mathrm{~N}$ is an integer-so that the smoothing curve is moved slightly. This is legitimate if the form of the statement of the criterion is changed slightly; the equal to or greater than statement is replaced by a greater than statement. We now say the probability of secing a failure is $\geq .90$ if the fraction defective $>.05$ (instead of $\geq$ ). In terms of the Gamma functions, we utilize "fractional defects" to achieve a "barely acceptable" fraction defective, then add one more defective unit.

\section{1-3 Discussion}

The above explanation was provided to answer repeated questions about the sampling criteria. However, as alluded to earlier, the real test of a sampling rationale for the Stockpile Evaluation Program is whether all defects are being detected by the kinds of tests utilized in the program, and whether they are found soon enough that major degradation of reliability or safety can be avoided (through corrective action). The answers to these questions are provided by examining the cumulative experience with stockpile weapons; the defects observed; the tests capable of discovering their presence; the rates of degradation of stockpiles because of these defects; the intensities of investigations to determine the causes of defects, their impacts on the stockpiles in which they were found, and corrective actions which will eliminate them from the stockpiles; and, finally, the promptness in accomplishing needed corrective actions.

Taking all of these things into consideration-a largely qualitative task, supplemented by numerous statistical evaluations of pieces and parts of the problem-we believe that the sampling quantities are adequate. One supporting indication is that the failure rates observed over the years are consistent with the Military Characteristics requirements; this indicates to us that the entire process of finding, investigating, and correcting defects throughout the weapon stockpiles works satisfactorily.

Further reductions in sample size appear unwarranted in light of the amount of test diversification we have found necessary to evaluate all aspects of weapon performance under all use conditions. The number of units allocaled to each of these different kinds of tests is already small; further reductions engender concern as to whether their individual frequencies would still 
be adequate. The relatively small numbers allocated to each of these tests, as compared to the total sample size, have been defended on the basis that the failure mechanisms they uniquely address-and which provide the reason for having that lype of test-were thought to represent small fractions of the failure mechanisms which existed, so that we could risk controlling them with fewer tests. However, recent studies suggest that "test unique" failure mechanisms are more prevalent than had been earlier supposed. Realistic appraisal of the program in light of this new information precludes additional reductions.

\section{1-4 References}

'Stockpile Evaluation Manual, published by Department 7260) (now 36x), February 1991.

'SAND8.3-1034, Determining Sample Sizes for Diversified Reliability Test Programs, F. W. Muiller, June 1984. 


\section{Topic V}

\section{PREDICTIONS INVOLVING SMALL PROBABILITIES (OCTOBER, 1974)}

The prediction of small probabilities often represents a misuse of mathematical models devised to represent real phenomena. The misuse of mathematical models is wide-spread, but is perhaps more serious in probability models. We will attempt to identify the problem by first treating limitations of mathematical models in general, and then discussing peculiarities of probability models.

\section{1-1 Mathematical Models}

It is generally understood that a mathematical model is intended to describe only the dominant catuses of a matural phenomenon, and that there are inevitably many less-significant caltuses that are not accounted for, but which prevent even the most useful models from being completely accuratc.

Our ability to model physical phenomena is closely likened to our ability to measure the caluses associated with them. To achieve more accuracy, measuring techniques must first be improved to measure causes previously treated as insignificant. Following the measurement of these caltses, they can be included in the model, and attempts at better control can begin. The state-of-the-art of measurement therefore determines, for any mathematical model, a level of accuracy which is within the bounds of propriety at a given time.

It is perhaps unfortunate, however, that there is never anything in the mathematical model, per se, that acknowledges any limitation in accuracy. Since only our considerable ability to calculate limits the precision of the results obtained from the model, there is often a confusion between precision and accuracy.

\section{1-2 Probability Models}

The confusion between precision and accuracy exists to some extent with any mathematical model, but is amplified in probability models by the peculiar difficulties encountered in measuring probabilities. To establish a probability one must measure the characteristics, not of a single experiment, but of an entire population of experiments, each a replication of the last. In these calses it is not so much the ability to measure that limits our accuracy but the need for so many measurements.

Because of this difficulty, our ability to measure probabilities lags far behind our ability (1) measure almost anything else. It is very rare that empirical evidence are available that will allow a statement of probability that is accurate to within one part in $10^{6}$. If a sufficient number of observations have been made, they almost certainly are a collection of observations from many dissimilar populations, so resulting probability statements do not apply to any real population, 
but instead to a fictitious composite population. The accuracy achieved in predictions made in this manner about any real population is extremely questionable. (This is one problem encountered in utilizing failure rate information available in many reliability handbooks. The accuracy of the failure rate information depends upon the structure or the composite population from which the data were collected. The more heterogeneous the subpopulations in the composite population, the poorer the accuracy of predictions about a real population. Of course, one often finds quotes of failure rates of one failure in $10^{8}$ or $10^{9}$ hours of operation.)

Because of the lack of accurate information about probabilities, analysts must apply considerable judgment in providing predictions about probabilities. The accuracy that may be attributed to the judgment of the analyst depends upon his experience, etc., but in any event is limited by the simple fact that human capabilities are limited. Accuracies of one in $10^{6}$ or more in probability statements are entirely heyond human capability or understanding, if not beyond human experience. Thus, we may say categorically that if there is no empirical evidence that will support an accuracy of $10^{\circ}$, then no such accuracy can be claimed.

Of course, it is not immediately clear that this is the entire story, since probability models are frequently devised that give the illusory appearance of breaking the problem into small pieces, where the accuracy required for each "piece" is not so prohibitive. ('onsider the following example.

If three things occurring jointly will bring about a particular result, we frequently devise a simple model to portray this situation by assuming the three events are statistically independent, and write

$$
\mathbf{P}(\text { result })=\mathbf{P}(\mathbf{A B C})=\mathbf{P}(\mathbf{A}) \cdot \mathbf{P}(\mathbf{B}) \cdot \mathbf{P}(\mathbf{C})
$$

where $P($ result $)$ is the probability of the joint occurrence of events $A, B$, and $C$, and $P(A), P(B)$, and $P(C)$ are marginal probabilities of the individual events.

Consider that we establish the probahilities of the individual events to be $10^{4}$ with a known accuracy of $\pm 10 \%$ (i.e., the range of each probability is from .0(0)(05 $10 .(0) 10.5)$. Are we justified in claiming that the probability of P(result) is $10^{4}$ with an accuracy of roughly $30 \%$ ? The answer is no! There are wo related reasons, both deriving from the limitations in mathematical models discussed earlier.

In the first place, a model set up to describe the fact that "A and $B$ and ( implies the result" (symbolized $A \cap B \cap C$.. result) cannot be automatically interpreted to mean "the result implies $A$ and $B$ and $C "$ (result . $A \cap B \cap C$ ). Yet our ascribing the stated accuracy to the above probability statement implies that this is the case. Of course, we would be content with our answer if we knew that all the other ways of obtaining the result were of insignificant probability. But what is or isn't significant in this case must be judged in comparison with $P(A B C)$, a probability evaluated thus far at $10^{\circ}$ ! (It doesn't help to observe that other ways of obtaining 
the result are insignificant by comparison to $\mathrm{P}(\mathrm{A})$, for example.) However, we have already admitted that such accuracies are beyond our measurement abilities and our judgment; we are left with the conclusion that no matter how long a list we compile of ways we can achieve the result, we cannot know that we have considered all the ways of achieving the result that have a significant probability when compared to numbers like $10^{9}$. In terms of the earlier discussion, we cannot know whether the model has accounted for the "dominant" causes of the phenomenon. To blindly trust such a model would be folly.

The other reason we cannot claim the accuracy stated is associated with the manner of combining the probabilities that we have at hand (the $P(A), P(B)$, and $P(C)$ ). In order to determine how these probabilities combine, we again find that we must measure probabilities on the order of $10^{\prime \prime}$, and this being the case, human judgment is unable to help. Were the probabilities of the result larger, human judgment could help somewhat by considering what things might have an influence on the way that events combine. For example, the assumption of statistical independence may be roughly translated into an assumption that only chance influences the way events are combined, and that no varying outside influences are present. These outside influences could be alnost anything, ranging from the fairly obvious influences of ambient environments to obscure and infrequent influences beyond our comprehension. Thus, assuming independence means that our model is based on the notion that the dominant influences do not vary.

Again, however, what is or isn't a dominant influence must be judged by considering its expected influence on the probabilities of the result. If the influence is almost never present (by comparison to the frequency of the result), or if its influence is very slight, an acceptable accuracy might be possible by just ignoring the influence. Hence, in the case of high probability results, we maly search for possible influences, and by controlling the dominant ones, justify the assumption of statistical independence for the purposes of calculating large probabilities.

When the probabilities become small, however, we are again at a loss in knowing what influences dominate; very rare influences can easily dominate a probability on the order of $10^{9}$. Searching for these obscure influences can take on all the aspects of a witch-hunt, with no guarantee that all the dominant influences will ever he found.

\section{1-3 Conclusions}

The above arguments raise serious questions about probability predictions for rare events. With such events there can be no certain knowledge that the dominating causes or influences have been accounted for in the model; hence objective agreement on results is not possible.

When an event should be considered to be rare is not always clear, but limitations in human comprehension strongly suggest that probabilities of $10^{-6}$ identify rare events. The potential for errors of omission in our probability models which predict probabilities of this size are simply too great for us to dare trust the predictions. 
This discussion is not intended to imply that we should not be concerned with events of low probability. If anything, we should be more concerned about them, as they usually arise in connection with events with intolerable consequences. That being the case, every reasonable effort should be made to assure that it will not happen. A search should be made for possible events that can produce the final event, and for possible factors that can influence the probabilities of these events. The system design can be adjusted to avoid the possibilities that are discovered, as long as it appears justified to accept the accompanying loss of other performance characteristics.

The point to be made is that although we can do a great deal to lessen a probability, we cannot predict the magnitude of the resulting probability in any meaningful way. 


\title{
Topic VI
}

\section{NOTES ON RELIABILITY ASSESSMENT (JANUARY 1974)}

\author{
Reliability - the probability a device behaves properly in use.
}

The reference to behavior emphasizes that a device reacts rather than acts. Behavior is the reaction or response to stimuli; whether a device does what it is supposed to do depends not only on the device, but also on the stimuli iexternal conditions, enviromments, and inputs) to which it is subjected.

An employment is the application of a set of stimuli to a device. It is analogous to the familiar trial or experiment in texts on probability and statistics, and is something that can be repeated, at least conceptually. Behavior is only observed in employments, so it is only when employments are known or stipulated that it makes sense to talk about behaviors and their probabilities, i.c., reliabilities.

Loosely speaking, probability can be considered to be the fraction of a population having a specified characteristic (e.g., bad, good, red, etc.). If, in a population of $\boldsymbol{N}$ members, $K$ members have a specified characteristic $\boldsymbol{E}$, then the probability that a randomly selected member from the population will have the characteristic $\mathrm{E}$ is $\mathrm{K} / \mathrm{N}$.

As a probability, reliability is also related to a population, but a population of what? Probability and statisties texts are replete with references to repeated trials involving the tossing of coins, rolling of dice, and drawing balls from urns. The populations implied there have as members employments consisting of the toss of a coin, roll of a die, etc. The term repeated trials suggests that the same coin would he involved in each employment, but one can also identify a population of employments in which many different coins are all tossed at once. These are different populations, and therefore may imply different probabilities. Variability between coins can affect the probability associated with the latter population, while the former will only reflect variability of the conditions from toss to toss.

It is necessary to have a definite population of employments in mind, even though replications may only be imaginary. Reliability, at Sandia, is generally defined for populations of "things" (e.g., weapon systems, components, etc.) whose behavior is of interest under variable conditions defined in a Stockpile-to-Target Sequence (STS). Becaluse of the variations in conditions or stimuli, any number of populations of employments may be envisioned involving the one population of "things;" for instance, each different temperature represents a different population of employments. To illustrate, each component might behave properly in tests under ambient conditions, yet fail if the temperature is too high (or low). Each different population of employments can have a different fraction of successful behaviors (i.e., reliability), depending on the test conditions (stimuli), ages of the "things," etc. 
Stimuli may be controllable or uncontrollable. With uncontrollable stimuli, it may only be hoped that their variations in test will approximate their variations in actual use, and that there is no correlation between them and the controllable stimuli. Possibly they are measurable so that they can be monitored for correlations.

On the other hand, controllable stimuli (location, time, temperature, etc.) can be prescribed and restricted to single values. The reliabilities for these populations of employments are easily envisioned. But how do these reliabilities relate to the reliability requirements which apply under any and all conditions specified in the STS? Here, the controllable stimuli may vary over wide ranges; e.g. temperatures may range from $-65^{\circ} \mathrm{F}$ to $165^{\circ} \mathrm{F}$. That is, the STS is a descriptive specification which does not restrict stimuli to single values. Different reliabilities could therefore exist for each of the different conditions allowed by the STS. For instance, visualize a plot of reliability versus some controllahle stimuli such as temperature.

If plots of reliabilities versus stimuli were available, and the frequencies of the various stimuli during use were known, then an average, or expected reliability can be obtained by weighting each reliability by the frequency of the defining stimuli. Mathematically, if the reliabilities, $r(x)$, are functions of the condition (stimuli), $x$, and if $f(x)$ describes the probahility of each condition in use' (not test), then

$$
R=\int_{a}^{b} r(x) d F(x)
$$

describes the expected reliability. $R$, where $a, b$ represent the range of conditions allowed in the STS.

If the probabilities of the various use conditions are not known, or knowable, as is the case, the expected reliability cannot be computed. DoD, however, assumes a distribution, and thus computes an expected reliability (or operational reliability). A general characteristic of this practice is that little weight or likelihood is given to the extreme environments in the STS where reliability is usually lowest. Consequently, DoD reliability statements may be considerably higher than they would be with a different probability distribution.

DOE has felt that the distribution of use conditions is, in large measure, determined by the strategies and counter-strategies of warring adversaries. These, of course, can be changed at will, making it very dangerous 10 rely on a given distribution of use patterns, particularly one that, ipso facto, allows a defense contractor 10 supply equipment that performs poorly under extreme conditions within the STS. If an antagonist devises a strategy which forces a use pattern dominantly in the extreme environments, the effectiveness of our weapons will be minimized. 
To avoid this trap, DOE assesses the lowest reliability throughout the entire STS. This does two things. First, this reliability will remain a lower bound on reliability, independent of any probability distribution on use conditions within the STS. Second, and perhaps more important, the emphasis on performance at the extremes of environment tends to force more design attention towards making devices impervious to the entire range of STS environments; usually the lowest reliability is not much lower than other points on the curve.

In those occasional instances where the lowest point on the curve is so low that requirements are not met, the range of use conditions is normally divided into sectors in which requirements are met and are not met. This identifies both the problems and the limitations that must be placed on use conditions to avoid the problems until corrective action can be taken.

Another facet of DOE reliability assessment should be mentioned before examining reliability modeling. The DOE assessments incorporate the effects of anticipated future degradation, and include assessments for limited life components at the end of their specified life. This means that the assessments are not intended to be descriptive of the reliability available today, but of the lowest reliability we anticipate during the stockpile life. Again, because of the emphasis on life considerations, it is usually the case that the lowest anticipated reliability is not significantly different from today's reliability. If the "end-of-life" reliability is too low to meet requirements, the time until requirements are no longer met can be identified. This identifies the problem and the time for incorporating corrective measures if a penalty in reliability is to be avoided.

\section{1-1 Reliability Modeling}

Reliability models simply relate component and part behaviors to system behaviors, where the term system merely describes the largest set of functional pieces of interest in a particular context.

Such modeling is necessary to apply the tremendous amount of test information that is available from component and subsystem tests. In fact, these tests constitute the main data base for assessment, with system test results basically serving as a control of the use of component data.

The alternative would be to perform myriads of tests of complete weapon systems in all the extreme use conditions; this would not only be exorbitantly expensive, but would delay the assessment process (components are available long before systems). DoD tends more towards this alternative, but are responding to less onerous requirements than DOE. Also, the lack of emphasis on environmental extremes permits testing under typical environments-a much simpler proposition. Finally, both the amount of component testing and DoD access to the results is limited by contractual arrangements between DoD and their contractors. However, even in the DoD, some modeling is necessary to address the continual changes in system designs. 
Some terminology is useful in discussing modeling.

A failure mechanism is the detailed, microscopic mechanism by which a failure can occur. It involves a single cause/effect chain. Typically the mechanism is associated with the hardware that fails, although other hardware or outside influences may be involved. (This fact is very important in determining which tests can detect the failure mechanism.)

Any assemblage of hardware (i.e., components, parts, subsystems, etc.) usually have a sizeable set of failure mechanisms associated with it. A failure event for this assemblage occurs if any of the individual failure mechanisms occur. Thus, a failure event is a macroscopic term representing the set of all appropriate failure mechanisms.

Systems can fail in different ways, earning different consequences (e.g., dud, abort, flare dud, etc.). When failure mechanisms are classified in these terms, reference is to failure modes. Failure modes, therefore, are also macroscopic terms, although they result in a finer division of failure mechanisms than loosely defined failure events.

Failure mechanisms, events, and modes can be identified in terms of basic sets of hehaviors. In the simplest case, a basic set will consist of two behaviors, failure or success. The behaviors in a basic set must be exhaustive and mutually exclusive, and each possible failure mechanism can belong to only one basic set.

A system is modeled as a collection of basic sets in such a way that the specification of one behavior from each basic set unambiguously determines the system behavior. For "exact" reliability equations, both success and failure events are included." However, by resorting to the use of approximate equations, it is almost always possible to develop equations which refer only to failure events. The approximation is excellent if the resulting system failure probability is small (less than .1), and is generally suitable for DOE needs.

In most reliability studies some sort of block diagram is used to display the logical relationships between the various failure events or basic sets within the system. Unfortunately, many of these diagrams suffer from a lack of standardization, short-comings in symbolism, and a desire to include too much information in the diagram. The result is often a hybrid diagram that is a combination functional schematic and logic diagram that defies unambiguous interpretation, except possibly by the person who prepared it.

For so-called Boolean Systems in which the components are considered to be either good or had, block diagrams can be drawn to display, unambiguously, the logical relationship hetween system and components. The block diagram can be interpreted as a switching circuit; component success is represented by a closed switch, failure by an open switch. System success amounts to finding at least one uninterrupted path from input to output. A component failure "cuts" the path through the component (opens the switch), and a system failure results when all paths have been "cut." 
Consider all the failure events within a system. Clearly, if all of these events occur, the system must fail; conversely, if none of them occur, the system must succeed. For all other sets of failure events, system failure or success depends on the system structure. A set of failure events that would result in system failure is referred to as a cut set. Consider the following three-component system example (Figure VI-1).

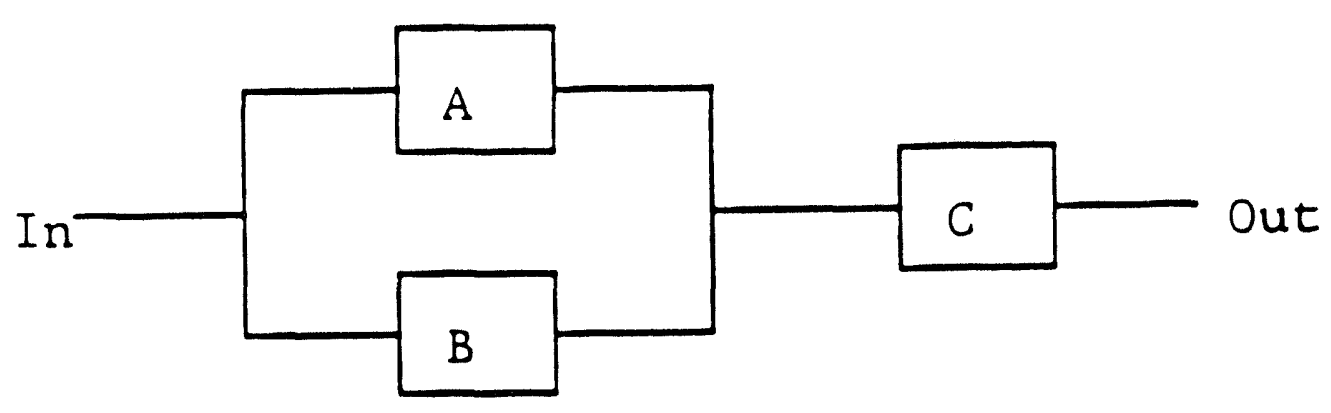

Figure VI-1. Three-Component System

The symbols $\boldsymbol{A}, \boldsymbol{B}$, and $\boldsymbol{C}$ refer to failure events or their probabilities. The cut sets are as follows.

\section{$\{A, B, C\},\{A, B\},\{A, C\},\{B, C\},\{C\}$}

A minimum cut set is a cut set in which all the failure events are essential, in the sense that no failure event can be removed from the set (changed to proper operation) without changing the system behavior from failure to success. The minimum cut sets are $\{A, B\}$ and $\{C\}$.

The approximate system failure probability equation is the summation of terms resulting from the minimum cut sets. A term for a given cut set is the product of the probabilities of the failure events in the set. In this case, the system failure probability, $Q$, is given by the following equation.

$$
\mathbf{Q} \approx \mathbf{C}+\mathbf{A B}
$$

If the probabilities of the failure events are $C=.001, A=B=.01$, then $Q \approx .0011$. (Given the correctness of assuming independence ${ }^{1,4}$ - which the above equation assumes-the true failure probability can be no larger than that computed by the approximate failure probability equation ${ }^{1.2,3}$.)

The approximate equation can also be directly interpreted as a logic equation, where the plus sign $(+)$ connotes a logical "or," and multiplication connotes a logical "and." Thus, logically, system failure, $Q$, occurs if $\boldsymbol{A}$ and $\boldsymbol{B}$ occur or if $\boldsymbol{C}$ occurs. 
Sensitivities of the system failure probability to a change in an individual failure event probability can be determined by examining only those minimum cut sets which contain that event. In most Sandia systems a failure event is either a first order event (it is a minimum cut set by itself) or it is a second order event (one other failure is in every minimum cut set it is in).

Any change, $\boldsymbol{C}$, in a first order event $\boldsymbol{C}$ produces an equal change in system failure probability, $\Delta \mathrm{Q}$. Since $\mathrm{Q}_{\text {old }}=\mathrm{C}+$ (other terms), then replacing $C$ by $\mathrm{C}+\Delta \mathrm{C}$ gives $\mathrm{Q}_{\mathrm{new}}=(\mathrm{C}$ $+\Delta \mathrm{C})+($ other terms $)$, and $\mathrm{Q}=\mathrm{Q}_{\text {new }}-\mathrm{Q}_{\text {old }}=\Delta \mathrm{C}$.

A change, $\boldsymbol{A}$, in a second order failure event $\boldsymbol{A}$, produces a change in the system failure probability that depends on the partial derivative of $Q$ with respect to $A$. If the partial derivative is not a function of $\boldsymbol{A}$, then

$$
\Delta \mathbf{Q}=\frac{\delta \mathbf{Q}}{\delta \mathbf{A}} \Delta \mathbf{A},
$$

where the partial is evaluated by substituting the original failure probabilities into it. For example, if

$$
\mathbf{Q}=\mathbf{A}(\mathbf{D}+\mathbf{F})+\mathbf{B}+\mathbf{C},
$$

then

$$
\delta \mathbf{Q} / \delta \mathbf{A}=\mathbf{D}+\mathbf{F}, \text { and } \quad \Delta \mathbf{Q}=(\mathbf{D}+\mathbf{F}) \Delta \mathbf{A}
$$

It will frequently occur that the same component is used more than once in a system, and that the same symbol is used for it whenever it appears. In this case, the partial derivative will be a function of that component probability. When this occurs, a correction factor of $(\Delta \mathrm{A})^{2}$ must be added to the above expression 2), so that you get the following equation.

$$
\Delta \mathbf{Q}=\delta \mathbf{Q} / \delta \mathbf{A} \Delta \mathbf{A}+(\Delta \mathbf{A})^{2}
$$

Again, the partial is evaluated in terms of the original failure probabilities. As an example, if

$$
Q \approx(A+B)^{2}+C
$$

then

$$
\delta \mathbf{Q} / \delta \mathbf{A}=\mathbf{2}(\mathbf{A}+\mathbf{B}),
$$


and

$$
\Delta Q \approx 2(A+B) \Delta A+(\Delta A)^{2}
$$

The need for the correction factor is easiiy verified by solving the relation

$$
\Delta Q \approx Q(A+A, \ldots) \cdot Q(A, \ldots)
$$

Note that the partials are completely determined by the cut sets which contain the event of interest.

\section{1-2 Reliability}

The definition of reliability refers to "proper behavior," implying interest in only two kinds of behavior, "proper" and "improper." The failure events and basic sets involve only a small number of discrete behaviors; in the Boolean Systems, only two. This is an obvious simplification; performance parameters of both systems and components normally are continuous variables; e.g., values of speed, altitude, voltage, resistance, elc., and are measured on continuous scales, There are several reasons why it is convenient, if not necessary, to dichotomize behaviors, an important one being the simplification of the basic sets. To do that, however, it is necessary 10 understand the relationship between continuous performance parameters, which reflect innumerable different hehaviors, and the discrete hehaviors utilized in reliability studies.

System requirements normally come in a dichotomized form in which a permissible range of values is specified for the parameters; e.g., bomb yield must be between two specified values, as must height of burst, weight, etc. The question is "how do these requirements relate to component parameters and basic set hehaviors?" The general situation can be illustrated with a simple, idealized "system" consisting of a battery, a discrete resistor, a "perfect" switch, and no battery internal resistance or circuit resistance. Suppose the system requirements are that the current (after the switch is elosed) shall be between 1.5 and 2.0 amperes for a period of time, and that power dissipated shall be between 20 and 30 watts.

The relationship between the system parameters and the performance parameters for the components can be discerned by modeling the physical parameters of the system. Ohm's law establishes the relationship between the system parameters (current and power) and the component parameters (battery voltage and resistance). The voltage-resistance (v,r) plot in Figure VI-2 identifies a "success" region where systems (i.e., voltage and resistance pairs) will produce the desired system behaviors; systems falling outside this region are failures.

If measurements of the voltages and resistances obtained from tests of individual systems were plotted on this diagram, a familiar "scatter diagram" should result. A system reliability requirement specifies the fraction of systems that should fall inside the success region. 


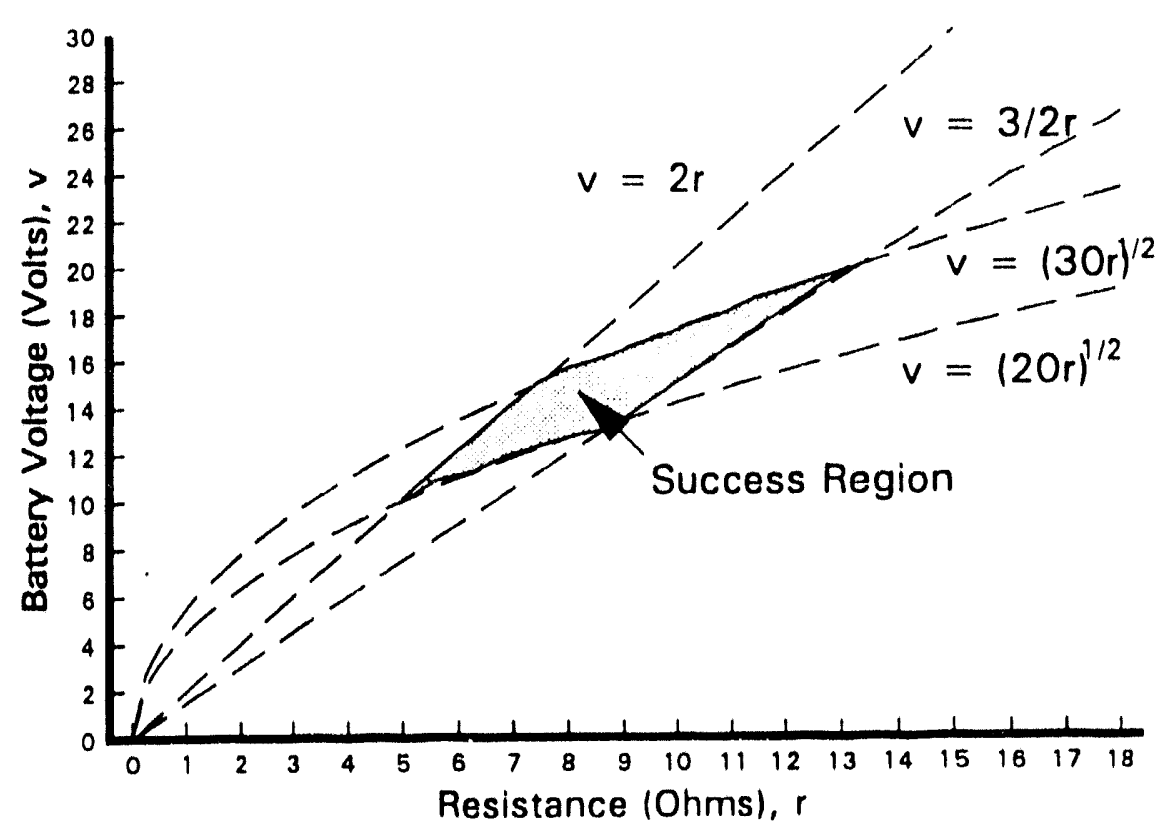

Figure VI-2. "Success" Regions

Even in this simple system, a significant characteristic of the general problem in defining success and failure of components is evident; component characteristics required to produce proper system behavior are strongly dependent on one another. In this system, the range of values of either voltage or resistance needed to produce a system success is strictly determined by the value of the other characteristic.

To keep the reliability model simple, and to satisfy the need in production, in order to maintain separate specifications for each component, a definition of each component's success and failure is developed that is independent of the other components. This, for the example system, corresponds to the intersection of two strips in the v,r plane, one strip being parallel to each axis (Figure VI-3).

Certain combinations of basic set behaviors are of interest. If both components are "good," the system is represented by a point in the rectangular intersection of the two strips; if one component is "good" and the other "bad," the point will be outside the rectangle, but within one of the strips; and if both components are "bad," the point will be outside both the rectangle and the strips.

The question that needs to be answered is "how do we accommodate the obvious differences in the two "success regions" identified in the two diagrams?" It is clear that there can be no equivalence between the definitions of success. However, by judicious choice of the ranges of component behaviors called "good," inferences about system behavior are possible. This can be done in several ways. 


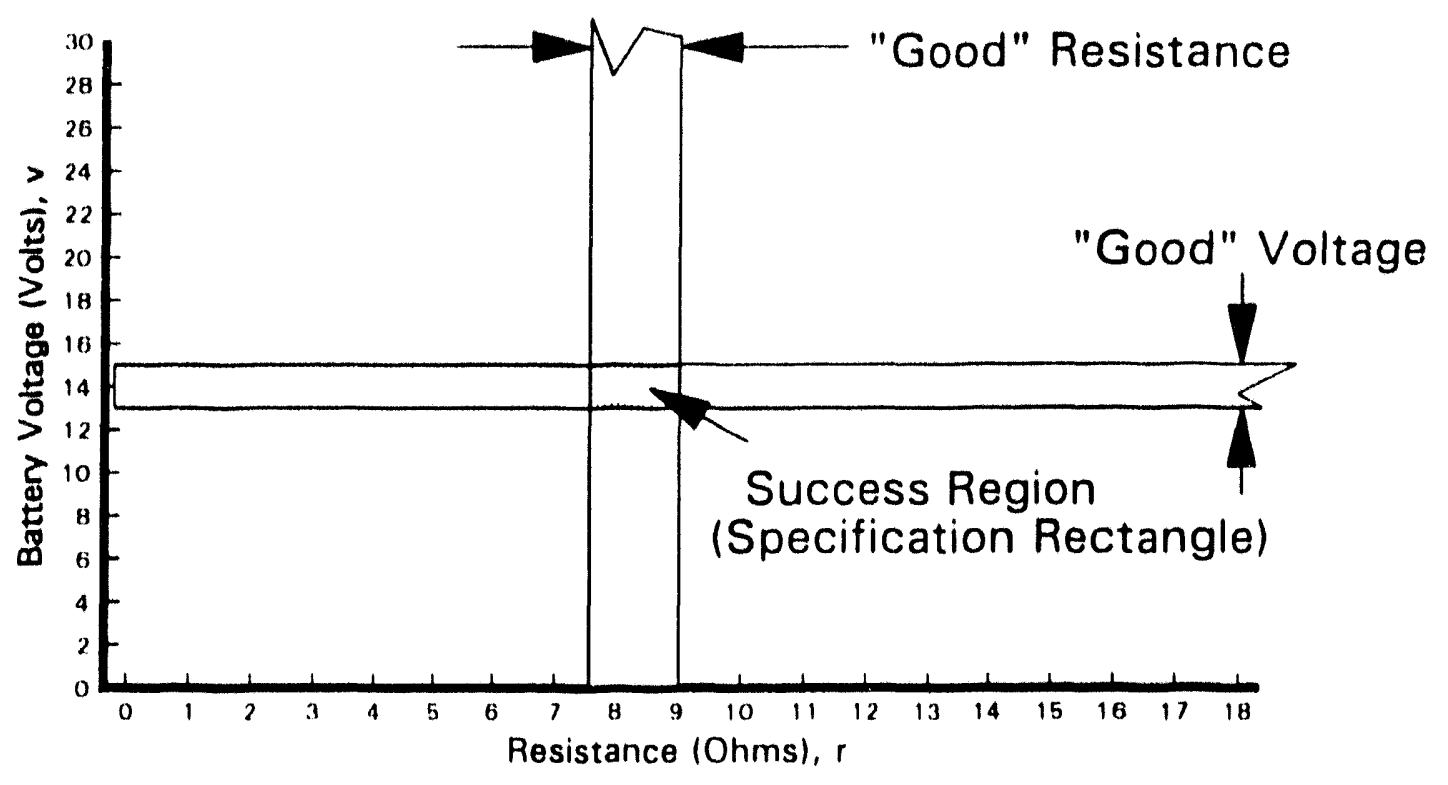

Figure VI-3. Success Region (Specification Rectangle)

First, by adjusting the specifications so the rectangular "success region" for components falls entirely within the system "success region," any system consisting of two "good" components will automatically meet system requirements. (Of course, the converse statement is not true; systems with "bad" components might still meet system requirements!) This relationship is depicted in Figure VI-4.

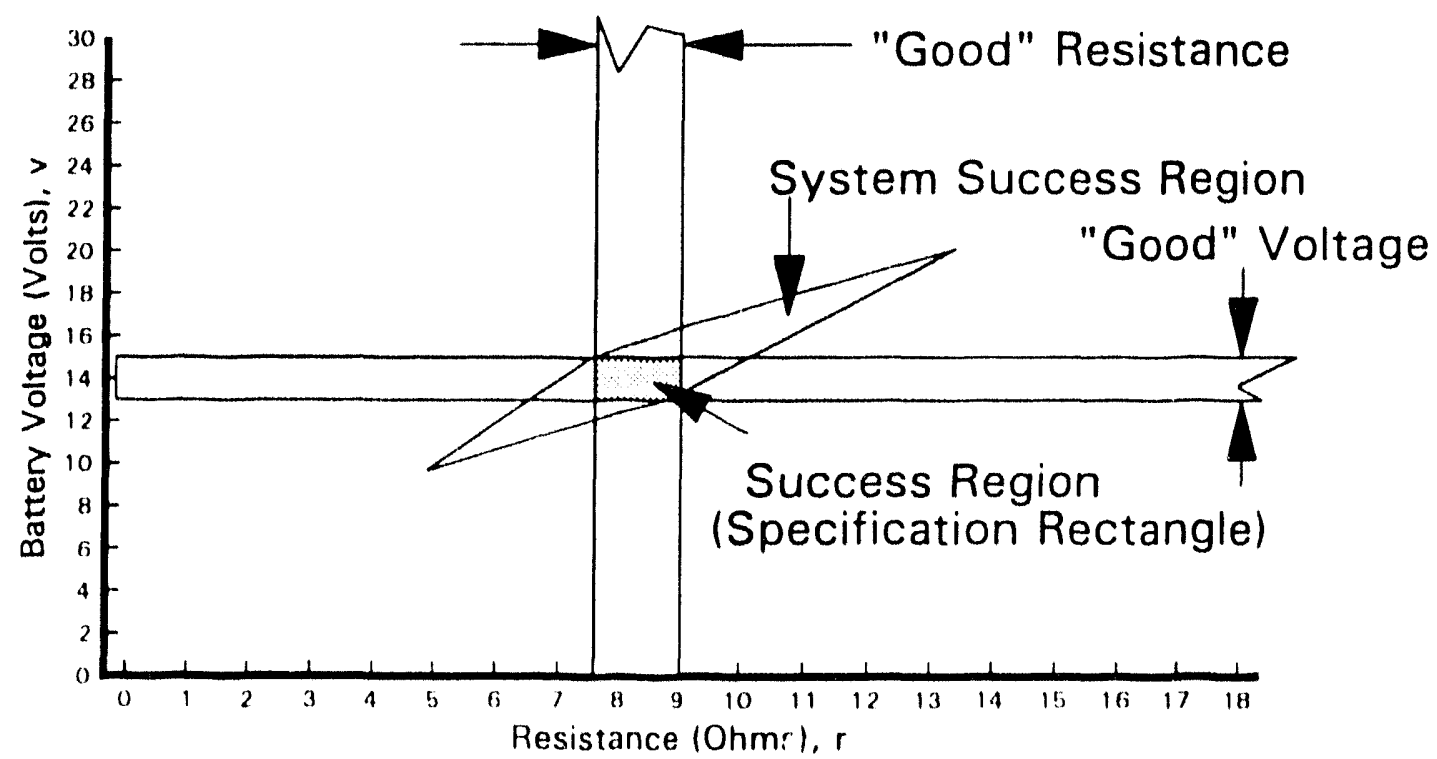

Figure VI-4. Specification Rectangle Established so that Component Success Implies System Success 
Since the system success region is larger than the "specification rectangle," the probability a randomly selected system will meet system requirements (i.e., perform reliably) is at least as high as the probability it will fall within the rectangle. Consequently, using component failure probabilities per their specifications to assess reliability will result in conservative assessments (failure probability possibly too high).

By adjusting the specification rectangle to include the entire system success region (see Figure VI-5), different inferences are possible. Now systems in which one or hoth components are "bad" will undoubtedly cause the system to be "bad." (Again the converse statement is not true; systems with "good" components may still result in system failure.) Assessments of system failure probability based on this definition of basic sets may be optimistic, i.e., too low.

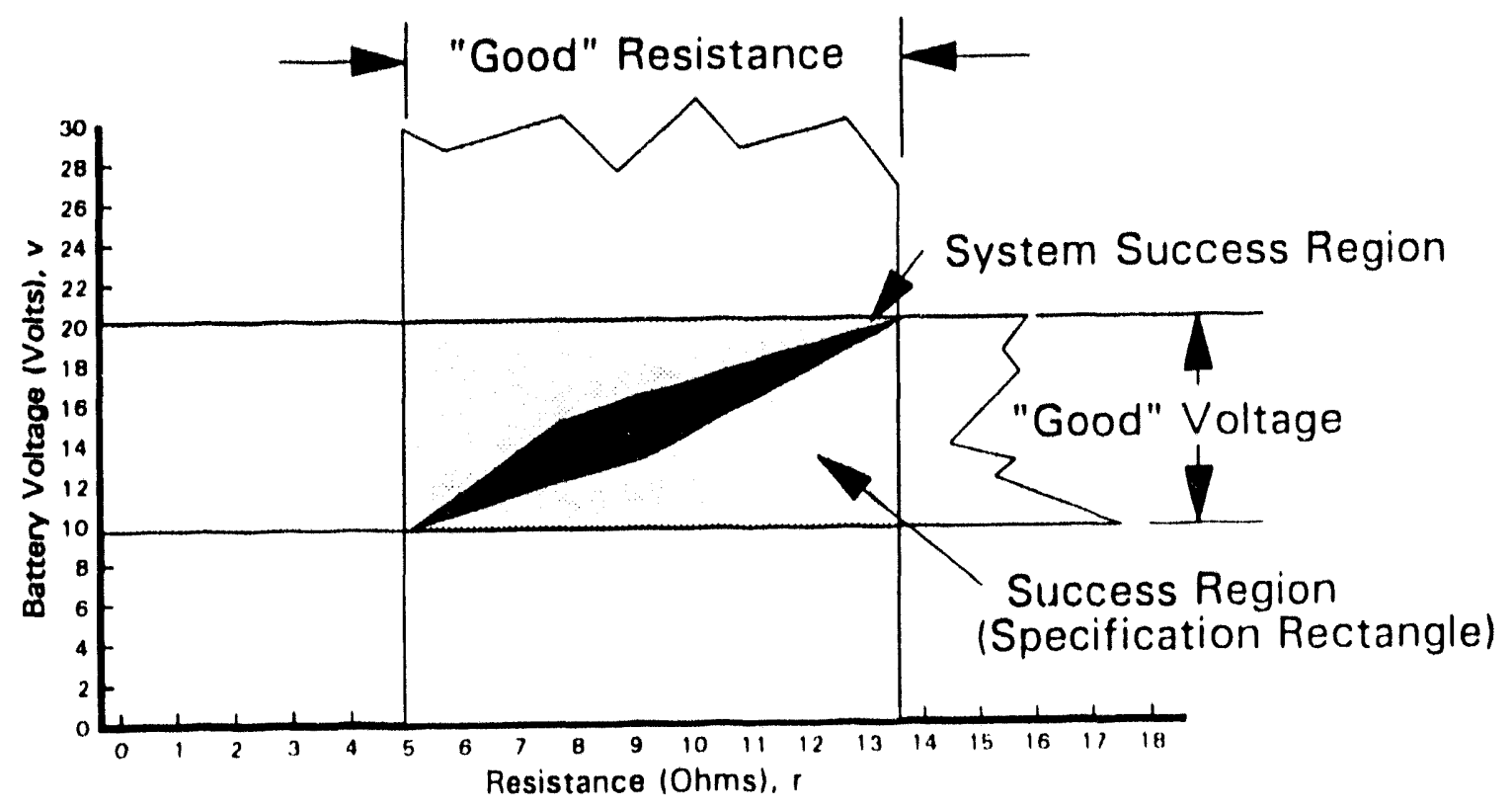

Figure VI-5. Specification Rectangle Established so that Component Failure Implies System Failure

By utilizing a dual specification system it is possible to assess upper and lower bounds for system failure probability. This establishes a solid relationship between what are frequently called "quality specification" and system reliability. However, notice that if the two bounds are significantly different, there is a large fraction of systems which fall inside the large rectangle, but outside the small one; such systems are deemed "marginal." and accurate assessment of reliability would require that the basic set definitions be abandoned, and a scatter diagram used to assess reliability.

There is another option. If the component failure probabilities (per the small rectangle) are small enough, the probability that both will fail can be neglected. The large rectangle can then be set so that if all components but one are "good," failure of that component implies system failure. A rectangle established this way is shown in Figure VI-6. 


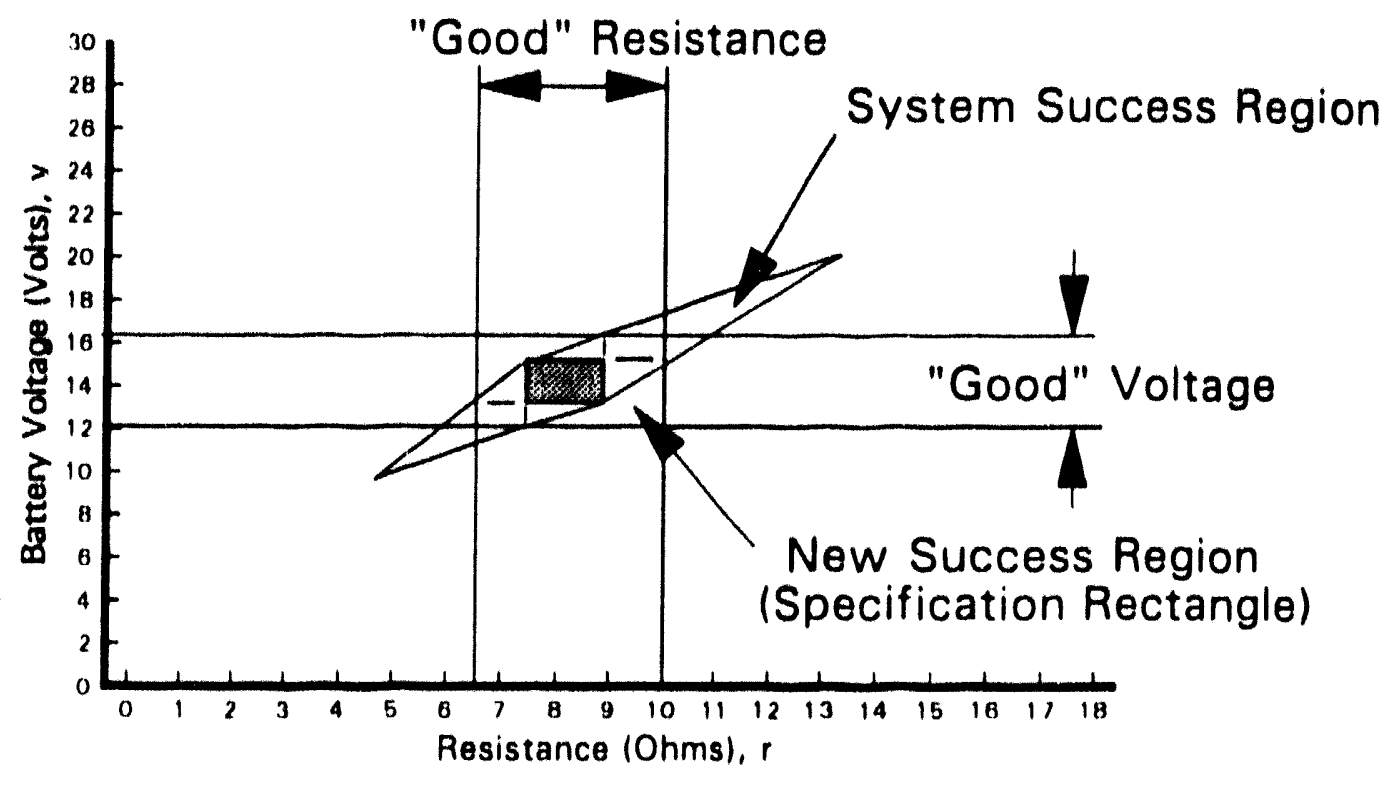

Figure VI. Specification Rectangle Established so that Component Fallure Implies System Failure with High Probability

Ideally, dual specifications developed this way could be used for all components. Test results could he reported as "go/no go" if the behaviors are inside the narrow range or outside the wide range; marginal behaviors could be reported as variables. Unfortunately, it is usually neither possible nor practical to identify the entire system success region. First, the dimensionality of the "hyper plane" in which the success region is defined increases with each additional cumponent specification, making visualization difficult. There may he hundreds of dimensions required to describe the success region for a real system. Then, because components in complex systems are highly interactive, the success region must be established empirically in a development test program. To explore the success region in all dimensions would be totally impractical for all but the simplest systems.

In these instances, the cost-effective approach is to explore the success region only enough to establish that a reasonable specification region (now a hyper-rectangle) is within the system success region, where reasonable means that production capability is not unduly taxed. If production is unable to meet the original specification, additional development testing can be done to attempt to enlarge the suecess region in the one or two troublesome dimensions, or an effort to improve the degree of control of production can be initiated. In all likelihood, all of these options will prove more practical than trying to identify the entire system success region.

\section{1-3 Assessing Reliability}

The prior sections discussed interpretations of reliability; developed rudimentary, but adequate equations for the probability of system failure; and identified how component behaviors 
relate to system behaviors. The last element of a reliability study is the assessment of component reliabilities.

Sandia advertises the use of all applicable data in assessing reliability. This sounds like "Motherhood," but places quite a strain on the word applicable. This section discusses what it means for datla to be applicable; in particular, it stresses that the mere statement that data are applicable does not all all indicate the manner in which they may be applied.

Assessment involves inferences of two types. The first is statistical inference, a relatively narrow form of inference, despite popular belief. When a random sample is taken from a population (of employments), proportions observed in the sample are taken as indicative of the proportions existing in the total population. Relating characteristics of random samples to population characteristics is statistical inference.

From earlier material it appears that statistical inferences about reliability could be made if employments involving the actual stockpile material were observed at the end of the material's stockpile life. However, sampling is seldom from the population (of employments) which corresponds to the lowest reliability. Neither are they conducted on material at the end of its life. Consequently, there are a number of differences between these employments and those observed in test programs. Tests vary in terms of the following.

a. Use conditions addressed (environments, delivery options, etc.)

b. Levels of assembly (component versus system, lab versus flight, etc.)

c. System configuration (fuzing option selected, launch platform, etc.)

d. Realism of hardware (non-WR hardware or hardware modified per safety or instrumentation requirements, etc.)

e. Realism of environmental simulation

f. Age of hardware

g. Sources of test material (sampling procedures)

h. Etc.

Tests under such different conditions can, at hest, be considered a motley collection of samples from different populations of employments. To relate the information gleaned from these tests to reliability requires a second, non-statistical kind of inference, often called inductive inference'. 
Assessments based on the assemblage of informallon from different kinds of tests will be in errer unless the differences belween the populattions of lest employments and the population of wors catse use employments do not alfece the probability of failure, of alfece it in a known way so suitable correction lictors call be applied. The potential for error is greatl, relying on "engineering judgment" 10 properly assess the impact of all physical phenomena. Too often wishful thinking is substituted for detailed andysis of cause/effect relationships, and oversights oceur frequently enough to warn off the dangers of complacency.

In general, statisties has little fo do with inductive inference. However, in questions about whether or not data from two or more different populations can be combined, statistics can offer some help. Significance tests which compare failure rates in the different populations will help avoid gross error in judging that the populations have the same failure rate.

Another saving feature lies in the chronology of testing. (ienerally, the more realistic tests end to oceur latter than those of less realism, where they serve as a check on the applicability of data from the latter, although sample sizes are seldom large enough to permit more than a gross check. The lesting performed as programs move into development, production, and stockpile tend 10 provide better and better checks of the applicability of earlier data. Assessment relies on this notion. For example, hefore development, only information from very broad experience with similar devices is alvailable. (ienerie failure rate data on transistors, gears. solder joints, etce are used to make predictions about lentative designs. Development provides an opportunity to identify failure mechanisms that might be peculiar to the present design, and to judge whether those predicted carlier are present. Predictions are adjusted aceordingly.

During production, tests are conducted on WR material; this is the first "direct" datat avalable, i.e., datat from the population of deviees that are being fielded. This testing not only provides a large yuantity of datia, but checks the validity (applicability) of the development and carlier lesting. If production lesting of WR hardware displayed failure mechanisms not experienced cartier, for example, the cartier datta would have to be discarded before an assessment is made. (Assessments begin when direct data are available.)

Laboratory system lesting provides a check of component testing: thight testing provides a check of lab system lesting: and so on. Bach test is performed in the expectation that data from it will he applicable, but later, more realistic lests maly show this to be wrong, resulting in data heing thrown out for assessment purposes.

An assessment is provided for the failure events in the basic sets, but it must be remembered that the fature events ate collections of failure mechanisms, each with its own caluse/effect chatin. This means that an assessment must actually deal with individual failure mechanisms, whose individual failure rates are summed to determine the failure rate for a failure event. 
Consider a single failure mechanism. Assessments for that mechanism should be based on tests where the entire cause/effect chain is present. Typically the associated component has been tested in development, production, and finally in the stockpile evaluation program, all of which include component, lab system, and flight testing. Perhaps, when considering different environments, options, etc., there are 10 or 12 different kinds of tests-different in ways that may or may not affect failure rate. In relation to the single failure mechanism of interest, there will be $2^{10}$ to $2^{12}$ different sets of data that can be assembled, only one of which correctly relates to the failure rate for that mechanism. (The combinations exist because each type of data can be included or excluded.)

In determining the proper data set, answers to the following types of questions must be obtained: Do development components or components of similar design have the same failure rate (under similar environments) as production material? Will components or systems in test exhibit the same faiiure rate in actual use? Will hehavior in one environment be the same as in another? Will aged material have the same failure rate as new material? And so on.

The individual failure mechanisms must be addressed in answering these questions, and the answers will vary from one failure mechanism to another (e.g., a "similar" component or a development component will have some failure mechanisms in common with the final component, but not all are likely to coincide. Design changes can eliminate some failure mechanisms present in development units and can introduce new ones at the same time. One environment may aggravate some failure mechanisms and not affect others; other environments may have just the opposite effect. Aging may manifest itself by increasing the frequency of one failure mechanism, but could conceivably lower the frequency of others, and so on.)

The identification and treatment of a failure mechanism is greatly enhanced if it occurs in a test and, through post mortem analysis, the precise failure mechanism (including its cause'effect chain) is identified. Armed with that information, it is relatively easy to determine which of the various kinds of tests can be used in assessment, i.e., determine in which tests the entire cause/effect chain is present.

Without precise knowledge of the failure mechanism and its cause/effect chain (the Achilles heel of the flight program because of the inability to perform post mortem analysis), the problem is more difficult. A flight failure could be the result of any of a number of failure mechanisms, all of which would have to be investigated and judgments made as to the likelihood of each having been responsible. Presumably, the more likely candidates would dominate the assessment, or, to be conservative, the worst case result can be used for the assessment.

With regard to conservatism, DOE advertises a conservative approach to assessment to the following extent. In cases where applicability of data from a given test is uncertain, strong justification would be required to treat the data in a way that would raise assessed reliability. That is, if data are available which would, if judged applicable, raise the assessed reliability, then the applicability of these data requires strong justification. Conversely, if data are available which would lower the assessed reliability, its exclusion would require strong justification. 
A final remark about assessment. Since assessment involves two kinds of inference (statistical and inductive), each with its own possibility of introducing error into the assessment, and since only errors from statistical inference can be determined, DOE does not provide statistical confidence statements for reliability; they imply (through the choice of terminology) that all uncertainties have been addressed.

\section{1-4 References}

'Müller, F. W., Elementary Reliability Technology, Iuly 1964 (Sandia Corporation Monograph SC-R-64-198).

${ }^{2}$ Birbaum, Z. W., Esary and Saunders, Multi-Component Systems and Structures and Their Reliability, Technometrics, Vol. 3, No.1, February 1961, pp. 55-77.

${ }^{3}$ Barlow, R. E., and Proshan, Mathematical Theory of Reliability, John Wiley \& Sons, 1965, pp. 202-208.

${ }^{4}$ Müller, F. W., and Ellefson, Simple Significance Tests for Independence of Events, Sandia Laboratories Report SLA-73-1041, December 1973.

${ }^{5}$ Muiller, F. W., Subsystem Requirements, Proceedings of 1970 Annual Symposium on Reliability, pp. 13-19. 
This page is intentionally left blank.

VI-16 
APPENDIX A

BIBLIOGRAPHY

A-1 
This page is intentionally left blank. 
Surveillance of the Future Stockpiles, SAND93-0999, May, 1993.

This SAND report contains a discussion of the preparations that are needed to conduct surveillance of nuclear weapons in a future where the production of new weapon types is unlikely.

Notable Historic Weapon Defects, SRD Report, SAND92-1937 RS7151/92/00022, November 1992.

Key weapon design defects found in stockpiled weapons over a period slightly exceeding 30 years are described. Reasons for the defects are given, as are the lessons learned.

Determining Sample Sizes for Diversified Reliability Test Programs, SAND83-1034, June 1984.

This paper develops criteria for planning test programs that consist of diversified types of tests.

Assuring Quality in Development and Design: An Antithetical Approach, Quality Progress, September 1984.

This paper advocates placing the primary control of quality of design and development activities in the hands of those involved in the activity, where quality techniques would also be used to control cost and schedule.

A Sample Preselection Process Designed to Enhance Early Planning Information, with F. W. Spencer, SAND81-1200, November 1981.

Early planning information about weapon samples can be provided without jeopardizing the necessary randomization of sample selection.

Quality Assurance at Sandia Laboratories, with S. L. Love, SAND78-0351, February 1978.

This SAND Report is a historical documentation of the Quality Assurance Program at Sandia.

A Study of the Nuclear Regulatory Commission Quality Assurance Program, with others, NUREG-0321, August 1977.

This report contains recommendations from a three month study of quality assurance in nuclear power plants as it is practiced in industry and regulated by the NRC.

Classical Upper Confidence Limits for the Failure Probability of Systems, with R. D. Halbgewachs \& R. C. Mueller, Research Report SLA-73-0563, September 1973. 
A computer code, CONLIM, is described which computes classical confidence limits for failure probability of systems based on component test results.

Simple Significance Tests for Independence of Events, with J. R. Ellefson, SLA-73-1041,

December 1973.

Exact significance tests are presented for use with system test results to check independence assumptions inherent in reliability assessment based on subsystem test results.

Maintaining a Limited Stockpile Without Spares or Rebuild Capability, with J. D. Deverman, SLA-73-1047, February 1974.

This report presents an alternative to usual reliability criteria when destructive testing would deplete the stockpile; testing is done to maximize the number of good weapons, based on testing to find defects that can then be corrected in the remaining weapons.

Subsystem Requirements, Proceedings of 1970 Annual Symposium on Reliability, pp. 13-19, 1970.

This paper addresses the difficulties of relating component and system requirements. (See Notes on Reliability Assessment, which incorporates these ideas.)

Survey of Techniques for Utilizing Hardware Redundancy, SC-TM-65-231, May 1965.

Basic features of techniques for utilizing redundant devices to improve reliability and safety are discussed.

Predictive Distributions, SC-RR-65-385, August 1965.

"Predictive" distributions are illustrated and proposed as a procedure to complement the application of confidence intervals and tolerance limits.

Tree Diagrams Applied in Analysis of Time Dependent System Failure Rates, SC-R-64-1376, November 1964.

The use of tree diagrams (developed in Elementary Reliability Technology) is extended to the analysis of systems in which the times of component failures are continuous random variables.

Elementary Reliability Technology, SC-R-64-198, July 1964.

A monograph detailing reliability modeling and assessment techniques for application to discrete systems such as weapons. 
Process Acceptance vs Lot Acceptance, with C. R. Clark, Proceedings of Eighth National Symposium on Reliability and Quality Control, 1960.

This paper presents and illustrates a philosophy of acceptance testing whose primary purpose is to evaluate and control the production process rather than accept or reject individual inspection lots. 
This page is intentionally left blank.

A-6 


\section{Distribution:}

Lawrence Livermore National Laboratory (2)

P. O. Box 808

Attn: L. M. MacLean, L-125

W. H. Hubbell, Jr., L-125

Livermore, CA 94550

Los Alamos National Laboratory (3)

P. O. Box 1663

Attn: T. Robinson, WX-5

W. H. Crismon, WX-5

R. L. Henderson, DRA-CDT

Los Alamos, NM 87545

U. S. Department of Energy

Albuquerque Operations Office

Attn: S. J. Guidice, Asst Mgr

R. Levine, WEB (5)

A. E. Whiteman, WQD

Albuquerque, NM 87115

25

300 R. L. Schwoebel

302 G. T. Merren

303 J. L. Duncan (6)

323 R. G. Easterling

324 V. J. Johnson

325 H. R. MacDougall

326 M. A. Blackledge

331 S. D. Spray

333 R. E. Smith

334 G. C. Novotny

335 J. M. Sjulin

361 R. F. Hahn
363

364

4100

4101

4102

4111

4111

4112

4114

4115

5000 R. L. Hagengruber

5002

5003

5004

5100

5104

5200

5202

5203

5204

5205

5300

5301

5302

5800

5801

5803

7141 Technical Library (5)

7151 Technical Publications

7613-2 Document Processing ior

DOE/OSTI (10)

8523-2 Central Technical Files

4111 F. W. Müller (3) 
This page is intentionally left blank. 

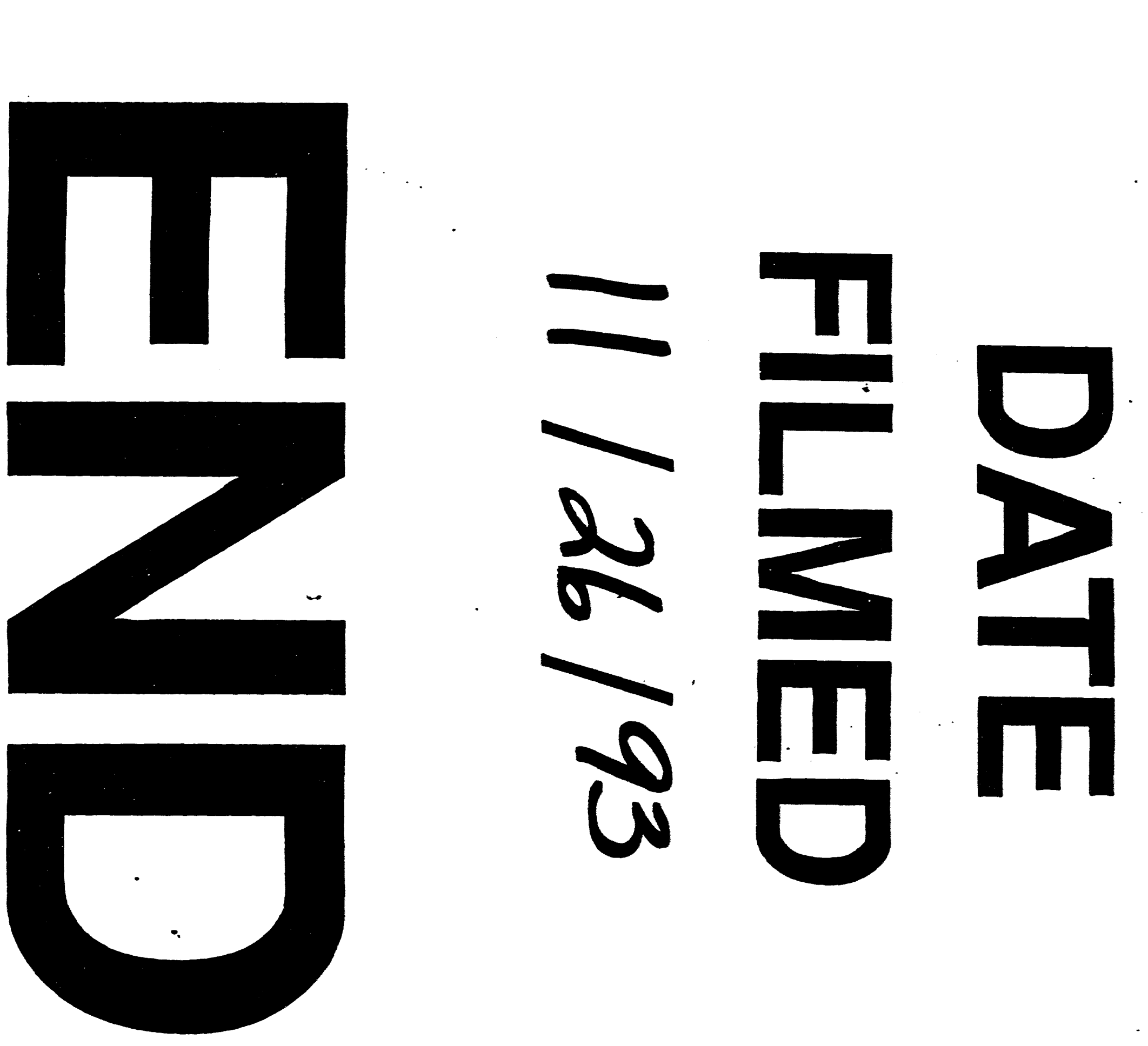
[

 